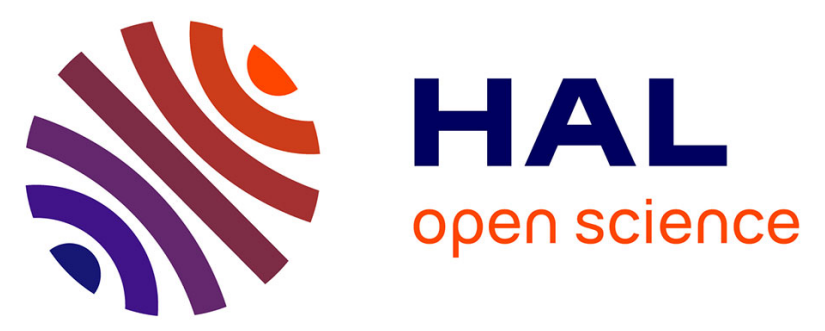

\title{
Importance of the nozzle-exit boundary-layer state in subsonic turbulent jets
}

Guillaume A Brès, Peter Jordan, Vincent Jaunet, Maxime Le Rallic, André Cavalieri, Aaron Towne, Sanjiva K. Lele, Tim Colonius, Oliver Schmidt

\section{To cite this version:}

Guillaume A Brès, Peter Jordan, Vincent Jaunet, Maxime Le Rallic, André Cavalieri, et al.. Importance of the nozzle-exit boundary-layer state in subsonic turbulent jets. Journal of Fluid Mechanics, 2018, 851, pp.83-124. 10.1017/jfm.2018.476 . hal-02378471

\section{HAL Id: hal-02378471 \\ https://hal.science/hal-02378471}

Submitted on 25 Nov 2019

HAL is a multi-disciplinary open access archive for the deposit and dissemination of scientific research documents, whether they are published or not. The documents may come from teaching and research institutions in France or abroad, or from public or private research centers.
L'archive ouverte pluridisciplinaire HAL, est destinée au dépôt et à la diffusion de documents scientifiques de niveau recherche, publiés ou non, émanant des établissements d'enseignement et de recherche français ou étrangers, des laboratoires publics ou privés. 


\title{
Importance of the nozzle-exit boundary-layer state in subsonic turbulent jets
}

\author{
Guillaume A. Brès ${ }^{1} \dagger$, Peter Jordan ${ }^{2}$, Vincent Jaunet ${ }^{2}$, Maxime Le \\ Rallic $^{2}$, André V. G. Cavalieri ${ }^{3}$, Aaron Towne ${ }^{4}$, Sanjiva K. \\ Lele $^{5}$, Tim Colonius ${ }^{6}$ and Oliver T. Schmidt ${ }^{6}$ \\ ${ }^{1}$ Cascade Technologies Inc., Palo Alto, CA 94303, USA \\ ${ }^{2}$ Institut PPRIME, CNRS-Université de Poitiers-ENSMA, Poitiers, France \\ ${ }^{3}$ Divisão de Engenharia Aeronáutica, Instituto Tecnológico de Aeronáutica, 12228-900 São \\ José dos Campos, SP, Brazil \\ ${ }^{4}$ Center for Turbulence Research, Stanford University, Stanford, CA 94305, USA \\ ${ }^{5}$ Dept. of Mechanical Engineering and Dept. of Aeronautics \& Astronautics, Stanford \\ University, Stanford, CA 94305, USA \\ ${ }^{6}$ Division of Engineering and Applied Science, California Institute of Technology, Pasadena, \\ CA 91125, USA
}

(Received ?; revised ?; accepted ?. - To be entered by editorial office)

To investigate the effects of the nozzle-exit conditions on jet flow and sound fields, large-eddy simulations of an isothermal Mach 0.9 jet issued from a convergentstraight nozzle are performed at diameter-based Reynolds number $1 \times 10^{6}$. The simulations feature near-wall adaptive mesh refinement, synthetic turbulence, and wall modelling inside the nozzle. This leads to fully turbulent nozzle-exit boundary layers and results in significant improvements for the flow-field and sound predictions, compared to those obtained from the typical approach based on laminar flow in the nozzle. The farfield pressure spectra for the turbulent jet match companion experimental measurements, which used a boundary layer trip to ensure a turbulent nozzle-exit boundary layer, to within $0.5 \mathrm{~dB}$ for all relevant angles and frequencies. By contrast, the initially laminar jet results in greater high-frequency noise. For both initially laminar and turbulent jets, decomposition of the radiated noise in azimuthal Fourier mode is performed and the results show similar azimuthal characteristics for the two jets. The axisymmetric mode is the dominant source of sound at the peak radiation angles and frequencies. The first three azimuthal modes recover more than $97 \%$ of the total acoustic energy at these angles and more than $65 \%$ (i.e., error less than $2 \mathrm{~dB}$ ) for all angles. For the main azimuthal modes, linear stability analysis of the near-nozzle mean-velocity profiles is conducted in both jets. The analysis suggests that the differences in radiated noise between the initially laminar and turbulent jets are related to the differences in growth rate of the Kelvin-Helmholtz mode in the near-nozzle region.

Key words:

\section{Introduction}

For jets, the state of the boundary layer at the nozzle exit is well recognized as an important parameter of the flow development and noise radiation. It has been the focus

$\dagger$ Email address for correspondence: gbres@cascadetechnologies.com 
of many experimental studies, including works from Bradshaw et al. (1964), Hill et al. (1976), Hussain \& Zedan (1978b,a), Husain \& Hussain (1979), Zaman (1985) and Bridges \& Hussain (1987), as well as more recent studies by Zaman (2012), Karon \& Ahuja (2013) and Fontaine et al. (2015). For full-scale nozzles at practical operating conditions, the nozzle-diameter based Reynolds number is $R e=\mathcal{O}\left(10^{7}\right)$, implying turbulent boundary layers and shear layers in the near-nozzle exit region. There is still debate about how thin the boundary layers are in a realistic engine context, as internal engine components will have non-trivial effects on the boundary layer development. On the other hand, in the context of idealized single-stream nozzles typically used for fundamental studies, these high-Reynolds-number features, important for the overall flow physics, remain challenging to capture.

In terms of numerical studies, various forms of the direct numerical simulation (DNS) and large-eddy simulation (LES) technique have been used over the years for jet flow and noise predictions. These research efforts have led to the availability of a substantial amount of data on compressible turbulent jets in general and, more specifically, on the influence of inflow conditions on the flow field and radiated noise. DNS studies can provide some valuable physics insights, but resolution requirements and computational costs restrict the simulations to Reynolds number of the order of $10^{3}$ to $10^{4}$ (Freund 2001; Suponitsky et al. 2010; Sandberg et al. 2012; Bühler et al. 2014a,b). The latter authors investigated Mach 0.9 laminar and turbulent nozzle-jet flows at $R e=18,100$ and proposed empirical scalings to account for the reduced Reynolds number and lower turbulence levels, and correct the sound predictions. As reviewed by Bodony \& Lele (2008), a larger body of work is available on large-eddy simulation of jet flows. In the early LES studies (e.g., Morris et al. 2002; Uzun et al. 2004; Bodony \& Lele 2005; Bogey \& Bailly 2005), the nozzle geometry was typically not considered and simulations relied on the introduction of disturbances at the inlet of the computation domain to force transition. To avoid the specification of tunable parameters for the forcing and the potential spurious noise associated with this, most recent simulations explicitly include a geometry at the inlet. However, inclusion of the physical geometry leads to challenges associated with the correct simulation of the boundary layers inside the nozzle. Turbulent boundary layers are difficult to resolve in LES due to the substantial cost of simulating the full range of flow scales that are present(Choi \& Moin 2012), to be added to the necessary cost of resolving the noise-source containing region at least 15 diameters downstream of the nozzle exit.

While early attempts were made to simulate initially turbulent jets (Uzun \& Hussaini 2007; Bogey et al. 2008), the computational expense of wall-resolved LES in the nozzle was prohibitive until recently. By now, Bogey \& Marsden (2016) performed simulation of a Mach 0.9 jet issued from a straight pipe nozzle at Reynolds number $2 \times 10^{5}$, which featured transitional turbulent nozzle-exit boundary layers, but this required a grid of $3.1 \times 10^{9}$ points. An early attempt at wall-modelled LES was made by Andersson et al. (2005), where the Reynolds number for an isothermal Mach 0.75 was increased from 50,000 to 900,000 , matching the value of a companion experiment. This was achieved on a structured grid of $30 \times 10^{6}$ points through the use of a wall function near the nozzle walls, though the grid was too coarse to resolve even the outer portion of the turbulent boundary layer in the nozzle.

Most of the current LES are therefore performed at reduced simulated Reynolds number, and the flow inside the nozzle is computed either through a coupling with Reynoldsaveraged Navier-Stokes (RANS) calculations (Shur et al. 2005a,b, 2011), or, more commonly, directly in the LES. In the latter case, the laminar flow issued from the nozzle 
mixes with the ambient fluid at the nozzle exit and quickly transitions to turbulence. However, in this situation, the laminar shear layers allow enhanced coherent shear flow dynamics in the transition region, which can lead to an increase in sound associated with the vortex roll-up and pairing process. In these cases, special treatment of the nozzle boundary layer is required, such as the introduction disturbances near the nozzle exit. Bogey and coworkers considered, in a series of papers, the role of inflow conditions and initial turbulence on subsonic jets originating from a straight cylindrical pipe at $R e=10^{5}$, with LES on structured grids with up to $255 \times 10^{6}$ points (Bogey \& Bailly 2010; Bogey et al. 2011, 2012). The initially laminar jet boundary layers were tripped inside the pipe, upstream of the nozzle exit, by adding either low-amplitude random pressure disturbances or low-level random vortical disturbances decorrelated in the azimuthal direction. The magnitude of the disturbances were empirically chosen to achieved targeted levels of peak turbulence intensity at the nozzle exit. Overall, they showed that these approaches weakened the coherent vortex pairing, increased the jet core length, and reduced the over-prediction of far-field noise spectra down towards those observed in experiments. As an alternative to this numerical forcing approach, Lorteau et al. (2015) used a geometrical tripping procedure (Pouangué et al. 2012) in the simulation of a Mach 0.7 jet at $R e=4 \times 10^{5}$ on a structured grid with $275 \times 10^{6}$ points. Vuillot et al. (2016) extended the approach to unstructured grids and simulated the same case on a $183 \times 10^{6}$ cell mesh. In both studies, the geometrical trip consisted of a small axisymmetric step added onto the surface inside the nozzle, reminiscent of boundary layer trip procedures used in experiments. It is argued that this method generates more natural turbulence, at the expense of additional constraints and tuning in the meshing process in order to robustly and efficiently integrate the added geometry with appropriate parameters. In terms of far-field noise, both LES studies showed that the geometrical trip was successful in reducing over-predicted spectra, within a few $\mathrm{dB}$ of experimental measurements.

The philosophy of the present study is to simulate the full scale system, including the turbulent flow inside the nozzle at the true Reynolds number and its effects on the nozzleexit boundary layer, on the flow-field in the jet plume and ultimately on the acoustic field, using a predictive LES approach. This is achieved by leveraging recently developed wall model, synthetic turbulence method, and localized adaptive grid refinement approach.

The manuscript is organized as follows. The experimental configuration and numerical setup are reviewed in $\S 2$, along with the modelling approaches used inside the nozzle (i.e., near-wall adaptive mesh refinement, synthetic turbulence seeding and wall modelling). Then, in $\S 3$, results from a series of preliminary large-eddy simulations are discussed to highlight the separate and combined effects of the different approaches used to simulate the internal nozzle flow. Further validation and analysis of the down-selected cases with initially laminar and fully turbulent nozzle-exit boundary layers are presented in $\S 4$, including azimuthal Fourier decomposition of the radiated sound. For the main azimuthal modes, linear stability analysis of the near-nozzle mean velocity profiles is conducted to investigate the differences in far-field noise between the two jets. Concluding remarks and future directions are discussed in $\S 5$.

\section{Flow configuration and numerical methods}

\subsection{Experimental setup}

The study focuses on isothermal subsonic jets issued from a round nozzle of exit diameter $D=50 \mathrm{~mm}$. The experiments were performed at the Bruit \& Vent jet-noise facility of 
the Institut PPRIME, Poitiers, France. Boundary layer transition inside the nozzle is forced using an azimuthally homogeneous carborundum strip of width $0.28 D$, whose downstream edge is located approximately $2.5 \mathrm{D}$ from the nozzle exit. The operating conditions are defined in terms of the nozzle pressure ratio $N P R=P_{t} / P_{\infty}=1.7$ and nozzle temperature ratio $N T R=T_{t} / T_{\infty}=1.15$. Here, the subscripts $t$ and $\infty$ refer to the stagnation (total) and free-stream (ambient) conditions, respectively. The jet is isothermal $\left(T_{j} / T_{\infty}=1.0\right)$, and the jet Mach number is $M_{j}=U_{j} / c_{j}=0.9$, where $U$ is the mean (time-averaged) jet exit streamwise velocity, $c$ is the speed of sound and the subscript $j$ refers to jet properties. With these conditions, the Reynolds number is $R e=\rho_{j} U_{j} D / \mu_{j} \approx 1 \times 10^{6}$.

Details about the nozzle geometry, experimental configuration and noise post-processing procedure are reported in Appendices A and B. Some of these details are also available in the supplemental materials, along with measurements and LES data.

\subsection{Numerical setup}

The present simulations use the LES framework developed at Cascade Technologies and leverage recent research efforts focused on modelling of the nozzle-interior turbulence (Brès et al. 2013, 2014). The framework is composed of the pre-processing meshadaptation tool "Adapt", the compressible flow solver "Charles," and post-processing tools for far-field noise predictions based on an efficient massively-parallel implementation of the frequency-domain permeable formulation (Lockard 2000) of the Ffowcs Williams \& Hawkings (1969) (FW-H) equation. Charles solves the spatially-filtered compressible Navier-Stokes equations on general unstructured grids using a densitybased finite-volume method. Time integration is explicit and uses the thirdorder TVD RK scheme of Gottlieb \& Shu (1998). The compressible fluxes are computed using a blend of central and upwind flux, resulting in a nominally 2nd-order scheme in space. The computational setup is briefly summarized below, with additional details about the solvers, the numerical schemes and the basic methodology available in Brès et al. (2017). Note that this reference does not cover the issues related to nozzle-interior turbulence modellings, which are the focus of the present work and are discussed in detail in what follows.

Schematics of the numerical setup are presented in figure 1, along with visualization of the instantaneous temperature and pressure field. The round nozzle geometry (with exit centered at $(x, r)=(0,0))$ is explicitly included in the axisymmetric computational domain, which extends from approximately $-10 D$ to $50 D$ in the streamwise $(x)$ direction and flares in the radial direction from $20 D$ to $40 D$. A very slow coflow at Mach number $M_{\infty}=0.009$ is imposed outside the nozzle in the simulation $\left(M_{\infty}=0\right.$ in the experiment), to prevent spurious recirculation and facilitate flow entrainment. All other simulation settings match the experimental operating conditions, including the Reynolds number. The Vreman (2004) sub-grid model is used to account for the physical effects of unresolved turbulence on the resolved flow, with constant coefficient set to the recommended value of $c=0.07$. A constant turbulent Prandtl number $P r_{t}=0.9$ is used to close the energy equation. To avoid spurious reflections at the downstream boundary of the computational domain, a damping function (Freund 1997; Mani 2012) is applied in the outflow buffer zone as a source term in the governing equations. In addition, the numerical operators are switched to lower-order dissipative discretization in the sponge zone for $x / D>31$ and $r / D>7$, to further damp turbulent structures and sound waves. Unless specified otherwise, all solid surfaces are treated as no-slip adiabatic walls.

In the preliminary parametric study, the far-field noise at $50 \mathrm{D}$ from the 


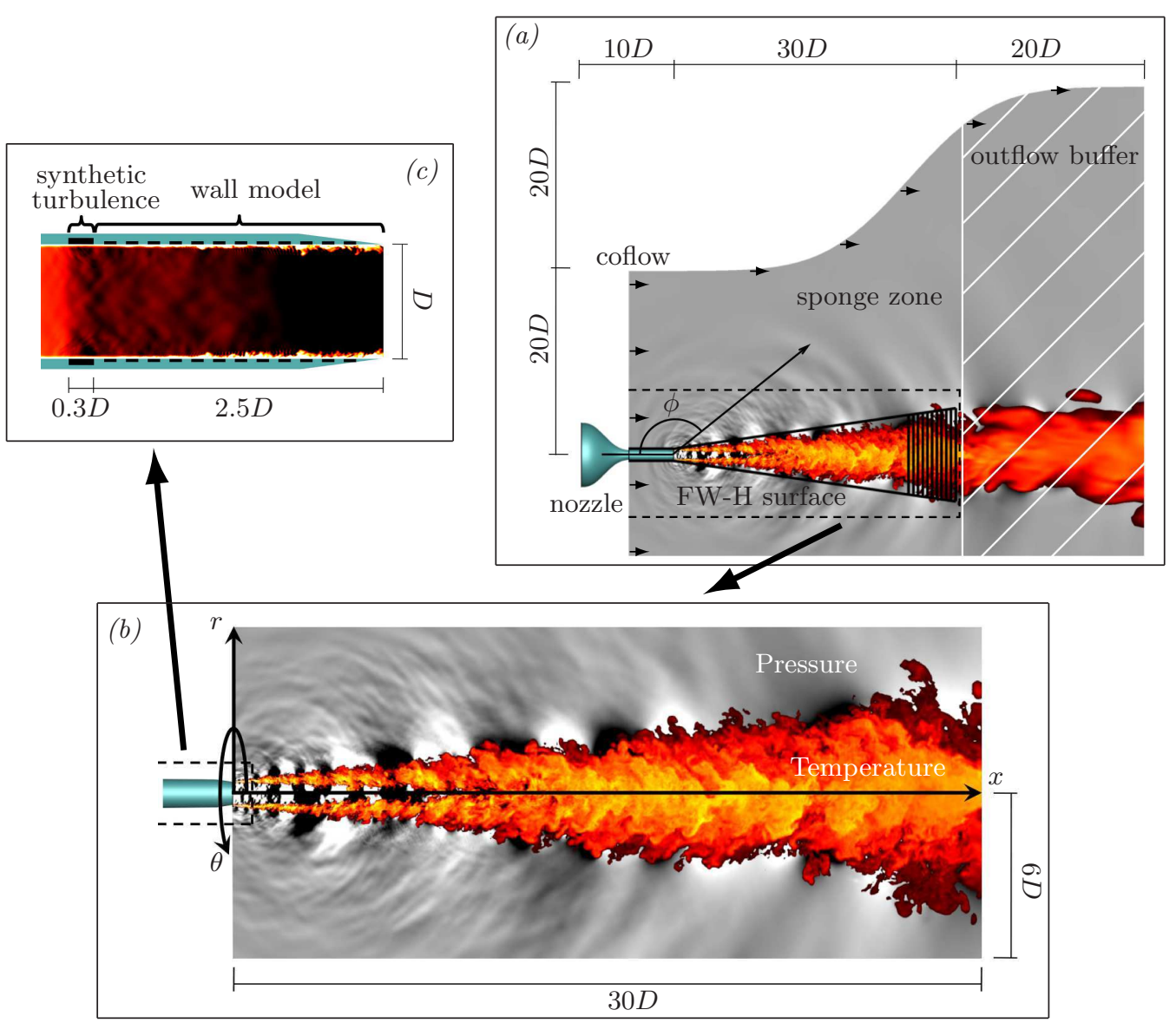

Figure 1. (Colour online) Schematics of the flow configuration and simulation setup: $(a)$ overview of the computational domain; (b) spatial extent of the LES database; $(c)$ modelling inside the nozzle.

nozzle exit was calculated for three different FW-H surfaces consisting of a cylindrical surface of radius $0.65 D$ up $x / D=0$, followed by a conical surface extending to $x / D=30$ with different spreading rates of $0.11,0.14$ and 0.17 . Here, the slopes are chosen based on estimates of the jet spreading rate (Zaman 1998, 1999). Similarly to previous studies (Brès et al. 2017), the results showed nearly identical spectra over the main frequency range for the three surfaces. The robustness of the prediction being thus confirmed, only the results from the intermediate surface outlined in black in figure 1(a) are reported. For treatment of the FW-H outflow disk, the method of "end-caps" of Shur et al. (2005a) is applied for $x \geqslant 25 D$, where the complex far-field pressure predicted from eleven FW-H surfaces with the same shape but outflow disks at different streamwise locations are phase-averaged. For all cases, the sampling period of the data recording on the FW-H surface is $\Delta t_{F W H} c_{\infty} / D=0.05$.

Table 1 lists the settings and parameters for each LES run considered, including the time step $d t$, the total simulation time $t_{\text {sim }}$ (after the initial transient is removed), and the data sampling period $\Delta t$ for the cases where the LES database of the full $3 \mathrm{D}$ flow field in primitive variable $(\rho, P, u, v, w)$ was collected, all expressed in acoustic time units (i.e., non-dimensionalized by $c_{\infty} / D$ ). The total computational cost is 


\begin{tabular}{|c|c|c|c|c|c|c|c|c|c|}
\hline Case name & $\begin{array}{c}\text { Mesh } \\
\text { size } \\
\left(10^{6} \mathrm{cv}\right)\end{array}$ & $\begin{array}{l}\text { refir } \\
\mathrm{BL}\end{array}$ & $\begin{array}{l}\text { yrid } \\
\text { Jement } \\
\text { Jet }\end{array}$ & $\begin{array}{c}\text { Synthetic } \\
\text { turbulence } \\
u_{\text {trip }}^{\prime} / u_{\tau}\end{array}$ & $\begin{array}{c}\text { Wall } \\
\text { model }\end{array}$ & $\frac{d t c_{\infty}}{D}$ & $\frac{t_{s i m} c_{\infty}}{D}$ & $\frac{\Delta t c_{\infty}}{D}$ & $\begin{array}{c}\mathrm{CPU} \\
\text { cost } \\
(\mathrm{kcore}-\mathrm{h})\end{array}$ \\
\hline \multicolumn{10}{|l|}{ Baseline LES } \\
\hline $10 \mathrm{M}$ & 10.8 & & & & & 0.001 & 2000 & & 40 \\
\hline $64 \mathrm{M}$ & 64.2 & & $x$ & & & 0.0005 & 600 & & 464 \\
\hline \multicolumn{10}{|c|}{ LES with nozzle-interior turbulence modelling } \\
\hline BL16M & 15.9 & $\times$ & & & & 0.001 & 600 & & 59 \\
\hline BL16M_Turb2 & 15.9 & $x$ & & 2 & & 0.001 & 600 & & 69 \\
\hline BL16M_Turb & 15.9 & $x$ & & 0.8 & & 0.001 & 600 & & 69 \\
\hline BL16M_WM & 15.9 & $x$ & & & $x$ & 0.001 & 600 & & 75 \\
\hline BL16M_WM_Turb2 & 15.9 & $x$ & & 2 & $x$ & 0.001 & 600 & & 81 \\
\hline BL16M_WM_Turb & 15.9 & $x$ & & 0.8 & $x$ & 0.001 & 2000 & 0.2 & 270 \\
\hline BL69M_WM_Turb & 69.0 & $x$ & $x$ & 0.8 & $x$ & 0.0005 & 1150 & 0.2 & 1514 \\
\hline
\end{tabular}

TABLE 1. Simulation parameters: synthetic turbulence amplitude $A=u_{\text {trip }}^{\prime} / u_{\tau}$, time step $d t$, total simulation time $t_{\text {sim }}$, and database sampling period $\Delta t$

also reported in thousand core-hours. All the calculations were carried out on the Cray XE6 system "Garnet" (Opteron 16C 2.5GHz processors, Cray Gemini interconnect, theoretical peak of 1.5 TFlop/s) on 1024 cores and 5152 cores for the standard and refined grids, respectively. The simulations with nozzle-interior turbulence modelling focused on adaptive isotropic mesh refinement of the internal boundary layer (prefix BL), synthetic turbulence (suffix Turb), and wall modelling inside the nozzle (suffix WM).

\subsection{Mesh adaptation and near-wall refinement}

The current meshing strategy has been used in previous jet studies (Brès et al. 2013, 2014, $2015,2016)$ and promotes grid isotropy in the acoustic source-containing region through the use of adaptive refinement. The starting point is a coarse structured cylindrical grid with a paved core and clustering of points in the radial direction at the nozzle walls and lip. The grid contains about 0.4 million purely-hexahedral control volumes. Several embedded zones of refinement with specific target length scale $\Delta$ are then defined by the user, and enforced iteratively by the adaptation tool, such that any cell with edge length (in any direction) greater than $\Delta$ will be refined in that direction, until the target length scale criterion is satisfied. The main refinement zone corresponds to the bulk of the mesh containing the jet plume, from $(x / D, r / D)=(0,1.5)$ to $(30,5)$, with $\Delta / D=0.14$. Then, within that zone, three additional conical refinement regions focusing on the jet potential core and surrounding the FW-H surface are defined, from the nozzle lip to $(x / D, r / D)=(10,2.5),(7.5,2)$ and $(5.5,1.5)$, with $\Delta / D=0.1,0.07$ and 0.04 , respectively. Finally, near the nozzle exit, three more refinement windows are centered on the lipline, extending to $x / D=2,0.7$ and 0.5 , with $\Delta / D=0.02,0.01$ and 0.0058 , respectively.

For the baseline cases, two grids were generated: a standard mesh containing approximately 10 million unstructured control volumes (cvs), and a refined mesh with 64 million cvs, by reducing the target length scale in half in each refinement zone in the jet plume. Note that for these cases, there is no specific near-wall or nozzle-interior refinement, and both grids have exactly the same coarse resolution inside the nozzle.

In contrast, for the simulations involving nozzle-interior turbulence modelling, it can 

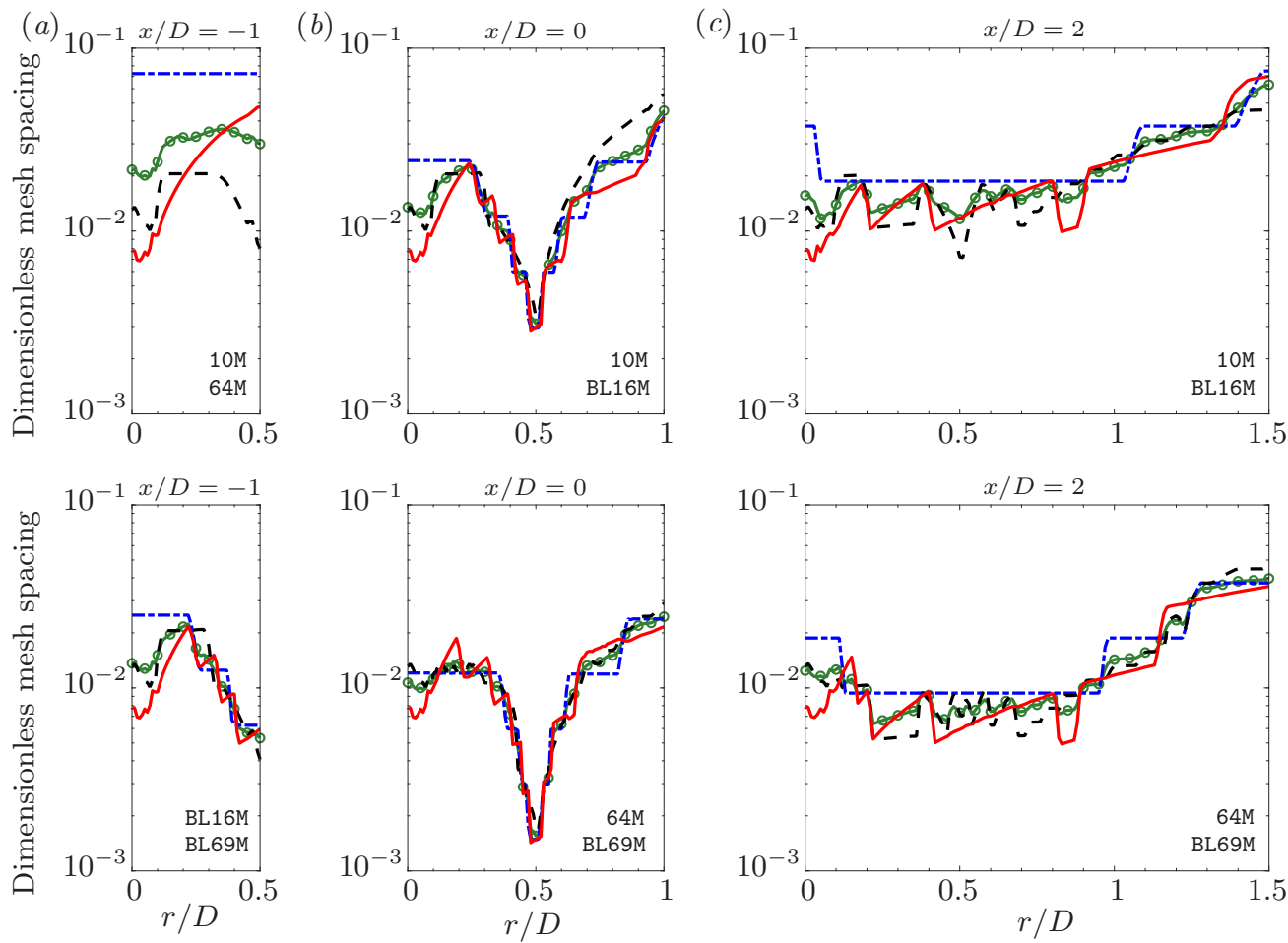

Figure 2. (Colour online) Dimensionless mesh spacing $(a)$ inside the nozzle at $x / D=-1,(b)$ at the nozzle exit $x / D=0$ and $(c)$ at $x / D=2$, in the axial $(\Delta x / D-\cdot-)$, radial $(\Delta r / D---)$, and azimuthal $(r \Delta \theta / D-)$ directions, and equivalent cell length $\left(\mathrm{vol}^{1 / 3} / D-\circ-\right)$ for the grid 10M (top) and BL69M (bottom). The grids with the same mesh spacing are also reported in the figures (see table 1).
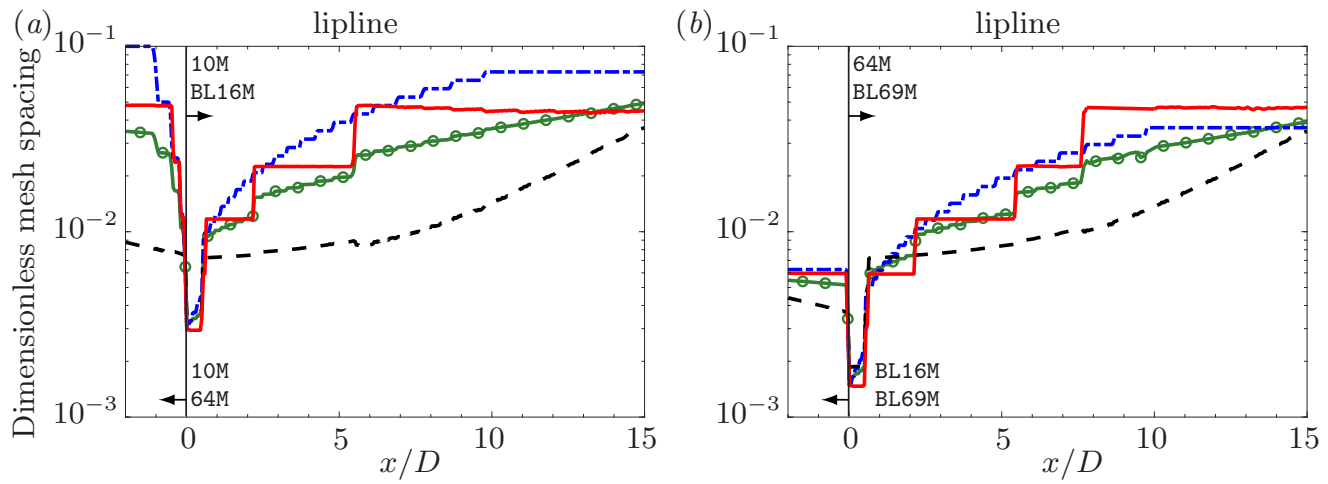

Figure 3. (Colour online) Dimensionless mesh spacing along the lipline at $r / D=0.5$, in the axial $(\Delta x / D--$ ) , radial $(\Delta r / D---)$, and azimuthal $(r \Delta \theta / D-)$ directions, and equivalent cell length $\left(v_{o l}^{1 / 3} / D-\circ-\right)$ for the grid $(a)$ 10M and (b) BL69M. The grids with the same mesh spacing are also reported in the figures (see table 1$)$.

be anticipated that further mesh refinement is needed inside the nozzle to resolve the large-scale three-dimensional turbulent structures associated with the internal boundary layers. Therefore, isotropic refinement is added to the previous adaptation strategy and applied from the start of the boundary layer trip at $x / D=-2.8$ to the nozzle exit at $x / D=0$, for a constant distance $0.085 D$ from the nozzle wall and with target length scale 
8

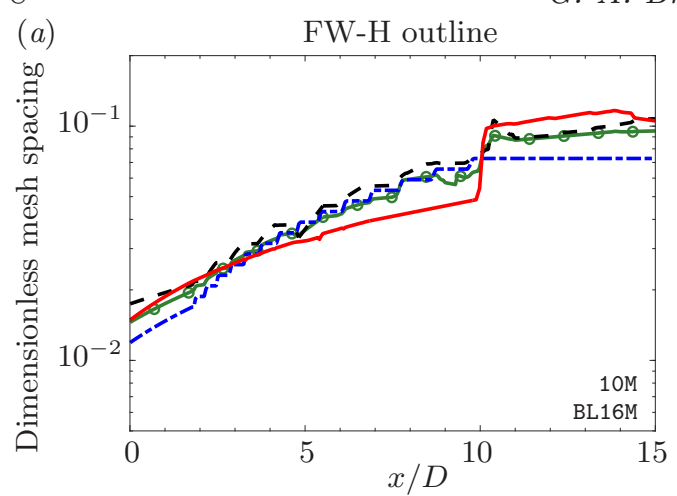

(b)

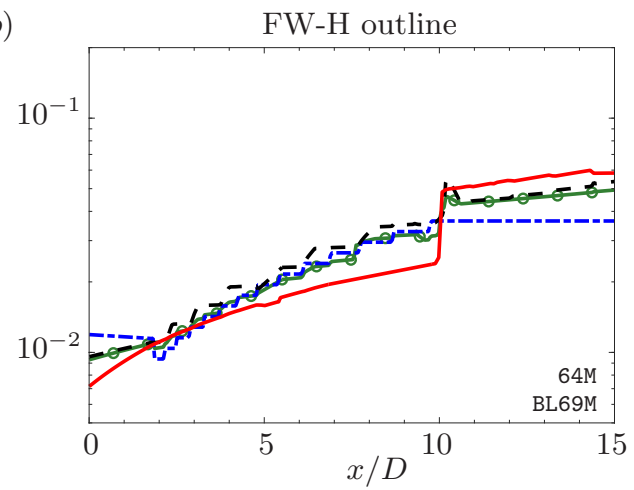

Figure 4. (Colour online) Dimensionless mesh spacing along the conical FW-H outline, in the axial $(\Delta x / D-\cdot-)$, radial $(\Delta r / D--)$, and azimuthal $(r \Delta \theta / D-)$ directions, and equivalent cell length $\left(\mathrm{vol}^{1 / 3} / D-\circ-\right)$ for the grid $(a)$ 10M and (b) BL69M. The grids with the same mesh spacing are also reported in the figures (see table 1).

$\Delta / D=0.0075$. The distance was chosen based on an initial estimate of the experimental nozzle-exit boundary layer thickness, $\delta^{99} / D \approx 0.08$, and the length scale was chosen to yield about 10-20 LES cells in the boundary layer. These choices lead to a finest wallnormal resolution of approximately $0.004 \mathrm{D}$, after adaption. As part of a preliminary study focusing solely on the flow inside the nozzle, additional simulations were performed on two grids where the target length scale for the near-wall refinement was reduced to 0.0058 and 0.0029 , respectively. Theses simulations yielded only limited improvements in the internal boundary layer predictions for a significant increase in computational cost. Therefore, we chose the more practical approach of keeping the resolution inside the nozzle on the modest side for wall-bounded flows. Mesh details at the nozzle wall and lip are reported in table 2 . The adapted grids with boundary layer refinement now contain approximately 16 million and 69 million cvs, for the standard and jet-plume refined cases, respectively.

Figures 2, 3 and 4 show, in logarithmic scales, the dimensionless mesh spacings for the four grids at different streamwise locations, along the lipline and along the outline of the conical section of the FW-H surface, respectively. In contrast to fully structured grids, the mesh length scales for the present unstructured grids with adaptation and hanging nodes are not globally predefined with smooth analytical form and vary in space depending on the refinement target length scales. The location of the user-defined grid transitions are clearly visible in the figures, in particular for the azimuthal length scale (red solid curve) in figure 3 at $x / D=0.5,2,5.5$, etc. . Nevertheless, the present isotropic refinement strategy leads to similar mesh spacing in all three axial, radial and azimuthal directions for most of the relevant regions of the computational domain. In terms of mesh isotropy, the only noticeable exception is near the lipline where the small radial resolution, present in the initial structured cylindrical grid to resolve the nozzle lip, remains in the adapted grids and leads to more anisotropy in the downstream region of the jet plume (see figure 3). The effect is however localized and overall, the cell aspect ratio (i.e., largest over smaller mesh length scale) is less than 2 for $85 \%$ (97\%) of the control volumes within the FW-H surface, for the grid without (with) jet plume refinement. The equivalent cell length $\mathrm{vol}^{1 / 3} / D$, which is the cubic-root of the cell volume, is therefore a representative metric of the resolution for the present isotropic hexahedral-dominant grids and is also presented in the figures. Following the analysis of Mendez et al. (2012) and Brès et al. (2017), this quantity 


\begin{tabular}{lllllll}
\hline \multirow{2}{*}{ Location } & Case prefix & $\Delta x / D$ & $\Delta r / D$ & $r \Delta \theta / D$ & vol $^{1 / 3} / D$ & $n_{\theta}$ \\
$\operatorname{trip}(x / D=-2.5)$ & 10M, 64M & 0.1000 & 0.0090 & 0.0478 & 0.0350 & 76 \\
& BL16M, BL69M & 0.0062 & 0.0045 & 0.0059 & 0.0055 & 530 \\
& 10M, BL16M & 0.0030 & 0.0034 & 0.0030 & 0.0031 & 1050 \\
nozzle lip $(x / D=0)$ & 64M, BL69M & 0.0015 & 0.0017 & 0.0015 & 0.0016 & 2095
\end{tabular}

TABLE 2. Representative mesh spacing at $r / D=0.5$ and corresponding number of grid points in the azimuthal direction $n_{\theta}$

is also used to estimate the limit Strouhal number $S t_{l i m}$ of acceptable resolution, corresponding to a wave resolved with eight grid point per wavelength: $S t_{\text {lim }}=D /\left(8 \mathrm{vol}^{1 / 3} M_{a}\right)$, where $M_{a}=U_{j} / c_{\infty}$ is the acoustic Mach number. Because the high-frequency noise sources are typically expected in the jet plume between the nozzle exit and the end of the potential (i.e., $0<x \lesssim 10$ ), the present grids are designed to approximately resolve the radiated noise spectra up to $S t_{\text {lim }} \approx 2$ for the standard mesh and to $S t_{\text {lim }} \approx 4$ for the refined mesh, based on the resolution on the FW-H surface in that region.

\subsection{Synthetic turbulence}

An extension to the digital filtering technique of Klein et al. (2003) was implemented for the generation of synthetic turbulence on unstructured grids, for both inflow boundary and wall boundary conditions. Because the turbulence levels inside the exhaust system upstream of the nozzle are typically unknown, the main objective of the synthetic turbulence is to seed the flow with fluctuations of reasonable amplitude, length and time scales, such that realistic turbulence is fully developed by the nozzle exit.

In the present work, synthetic-turbulence boundary conditions are used to model the boundary layer trip present in the experiment at $-2.8<x / D<-2.5$ on the internal nozzle surface (see figure $1(c)$ ). Based on the initial estimate of the experimental nozzleexit boundary layer thickness, the trip is therefore located more than $30 \delta^{99} / D$ from the nozzle exit, which is sufficient for the spatial development of a turbulent boundary layer.

The wall friction velocity $u_{\tau}$ is often used as a scaling parameter for the fluctuating component of velocity in wall-bounded turbulent flows. An initial value for $u_{\tau}$ was estimated based on the average wall shear stress downstream of the trip location for preliminary simulation on the $16 \mathrm{M}$ mesh. Fluctuations are then introduced in each component of the zero-mean velocity field at the wall boundary faces of the trip, with a prescribed amplitude $u_{\text {trip }}^{\prime}=A u_{\tau} / \sqrt{3}$. As part of the initial parametric studies, two different amplitudes, $A=0.8$ and 2 , were used, with the former value applied for most of the computations. For all cases, $\Delta_{\max }$ and $2 \Delta_{\max } / u_{\text {trip }}^{\prime}$ are used as initial estimates of the length and time scales of the input fluctuations, where $\Delta_{\max }=\max (\Delta x, \Delta r, r \Delta \theta)$ is the largest mesh spacing at the location of the trip (see table 2). Physically, this can be interpreted as the introduction of isotropic eddies of turbulent kinetic energy $1 / 2\left(A u_{\tau}\right)^{2}$ and dimensions comparable to the local mesh size. Here, the chosen length scale is also similar to the thickness of the experimental trip.

While the present work focuses on the $M_{j}=0.9$ case, different Mach number conditions ranging from 0.4 to 0.9 were considered as part of a broader LES study and nearly identical initial estimates $u_{\tau} / U_{j} \approx 0.042$ were obtained in all cases. Similar values can be obtained using simple flat-plate zero-pressure-gradient turbulent boundary layer approximations. Assuming the classical form of the skin-friction coefficient in turbulent flows, 
$c_{f}=0.0576 R e_{x}^{-1 / 5}$, with $x$ as the distance between the start of the straight section of the nozzle and the boundary layer trip, the empirical value of the wall friction velocity at the trip would be between $u_{\tau} / U_{j} \approx 0.041$ and 0.044 for $M_{j}=0.9$ to 0.4 . These estimates further confirmed the choice of order-of-magnitude for the coefficient $A=\mathcal{O}(1)$.

\subsection{Wall modelling}

When active, the equilibrium wall model, based on the work of Kawai \& Larsson (2012) and Bodart \& Larsson (2011), is applied inside the nozzle in the straight-pipe section between the boundary layer trip and the nozzle exit (see figure $1(c)$ ). The present method falls in the category of the wall-stress modelling approach (see reviews by Piomelli \& Balaras 2002; Larsson et al. 2016): unlike hybrid RANS/LES and detached-eddy simulations (Spalart 2009) that solve the unsteady Navier-Stokes equations on a single grid, with a RANS model near the wall and a LES model in the rest of the domain, the unstructured LES grid is formally defined as extending all the way to the wall (i.e., identical to a simulation without wall model), and a separate (structured) grid is embedded near the wall to solve the 1D RANS equations. The RANS solver takes information from the computed LES flow-field a few cells away from the wall, and returns back the shear stress $\tau_{w}$ and the heat transfer $q_{w}$ at the wall, to be used as boundary conditions for the LES wall-flux computation.

For most convex surfaces, the RANS grid is a simple extrusion of the wall surface mesh along the normal vector of each wall face. Following the recommendations of Kawai \& Larsson (2012), the wall-model-layer thickness (i.e., the distance from the wall where the RANS solver takes the LES information) is set to at least three LES cells away from the wall. In previous work (Brès et al. 2013), various sizes and stretching coefficients were considered for the inner-layer RANS grid and the default values of 40 cells and $10 \%$ stretching are used in the present study, for a wall-normal grid spacing in wall units $y_{R A N S}^{+}=\mathcal{O}(1)$. As shown in table 1, for the present cases with no specific attempt to optimize the performances, the extra computational cost of the wall model is about $27 \%$ of the standalone LES cost, similar to the value of $30 \%$ reported by Bodart \& Larsson (2011). Load-balancing of the wall-model procedure has been suggested as an approach to potentially reduce this additional cost.

\section{Parametric study of nozzle-interior turbulence modelling}

First, a study of the separate and combined effects of near-wall adaptive mesh refinement, the introduction of synthetic turbulence, and wall modelling is conducted on the standard mesh. To provide consistent comparisons, the same total simulation time $t_{\text {sim }} D / c_{\infty}=600$ is used for the computation of the flow statistics and far-field noise spectra presented in this section. Down-selected cases are then simulated for an extended period and discussed in $\S 4$.

\subsection{Effects of nozzle-interior turbulence modelling on flow field results}

\subsubsection{Instantaneous flow field}

Figure 5 shows the instantaneous flow inside the nozzle for the various cases with and without nozzle-interior turbulence modelling. Recall that both baseline cases 10M and 64M have the same operating conditions and same coarse mesh inside the nozzle. This leads to the same internal flow field and thin laminar boundary layer, with no visible velocity fluctuations inside the nozzle (see top row in figure 5).

In terms of the effects of the models, the first conclusion is that the mesh adaptation has 
(a)
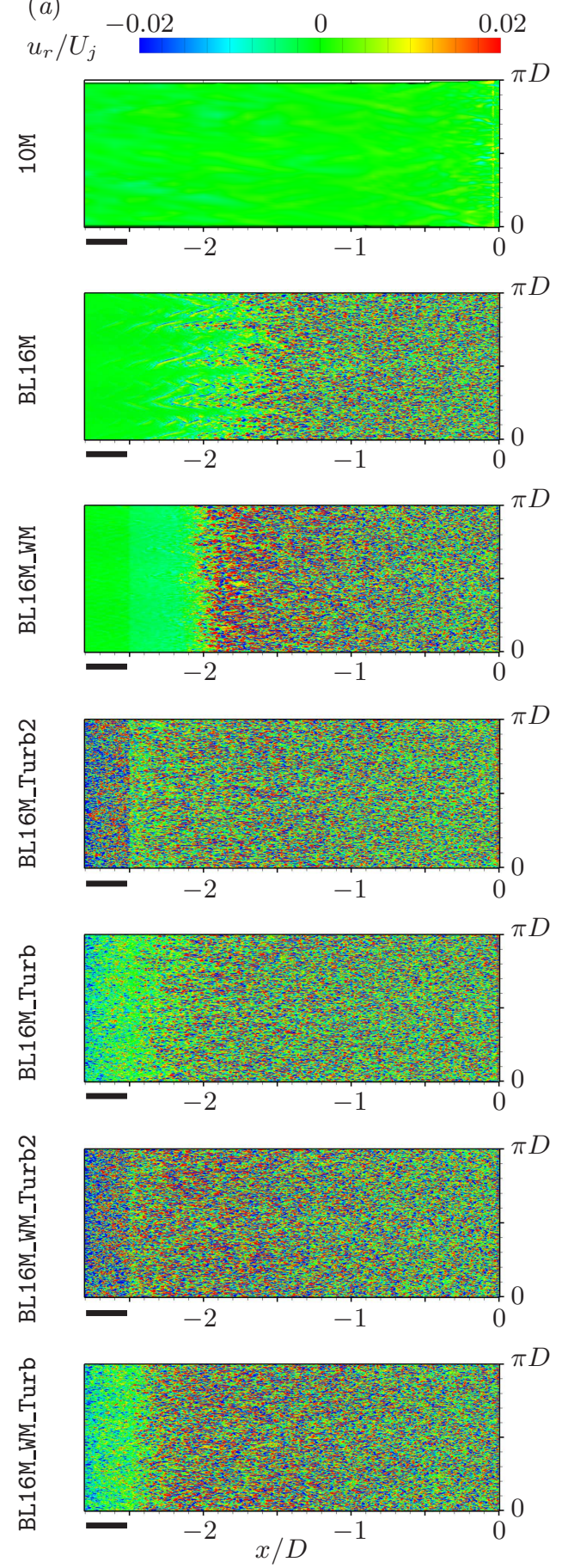

(b)
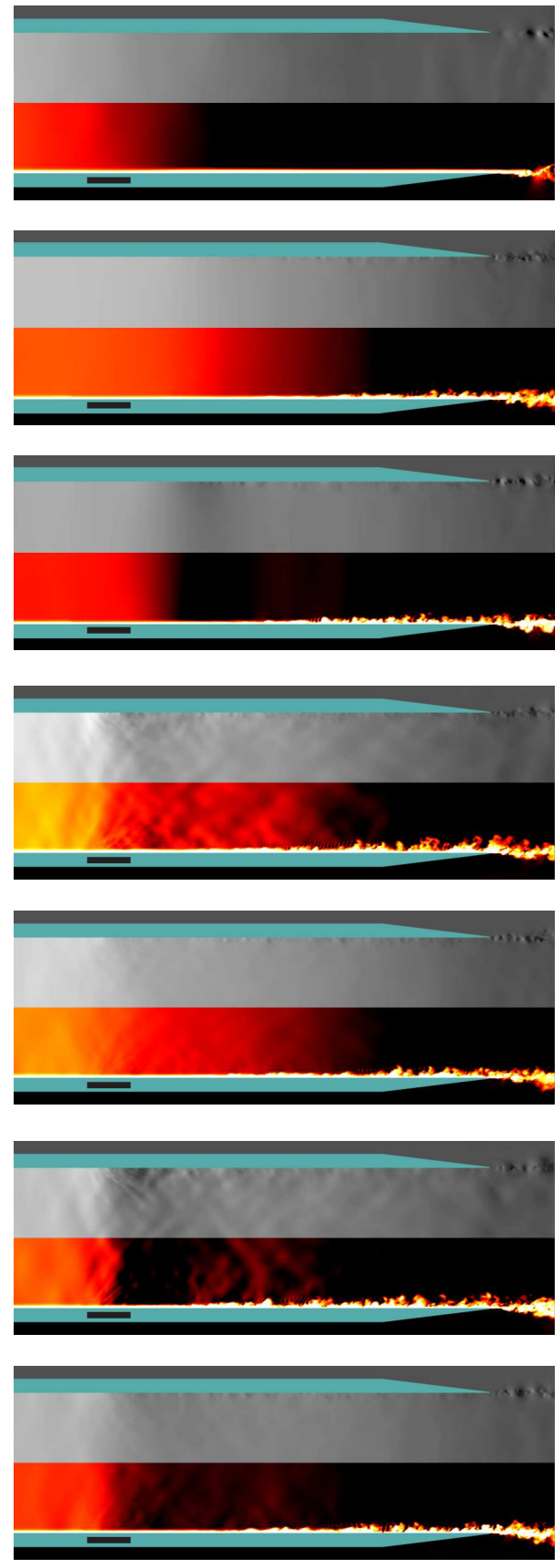

Figure 5. (Colour online) Instantaneous flow field inside the nozzle, for the baseline LES 10M (top row) and LES with nozzle-interior turbulence modelling: (a) Wall-normal velocity $u_{r} / U_{j}$ in the first cell near the (unrolled) nozzle interior surface. When active, the synthetic turbulence is applied for $-2.8<x / D<-2.5$ ( $)$ ); (b) pressure (top half - grey scale) and temperature field (bottom half - red scale) in the mid-section plane inside the nozzle. The colour ranges are $-0.1 \leqslant\left(P-P_{\infty}\right) / P_{\infty} \leqslant 0.2$ (black to white) and $1.0 \leqslant T / T_{\infty} \leqslant 1.05$ (black to light yellow). 

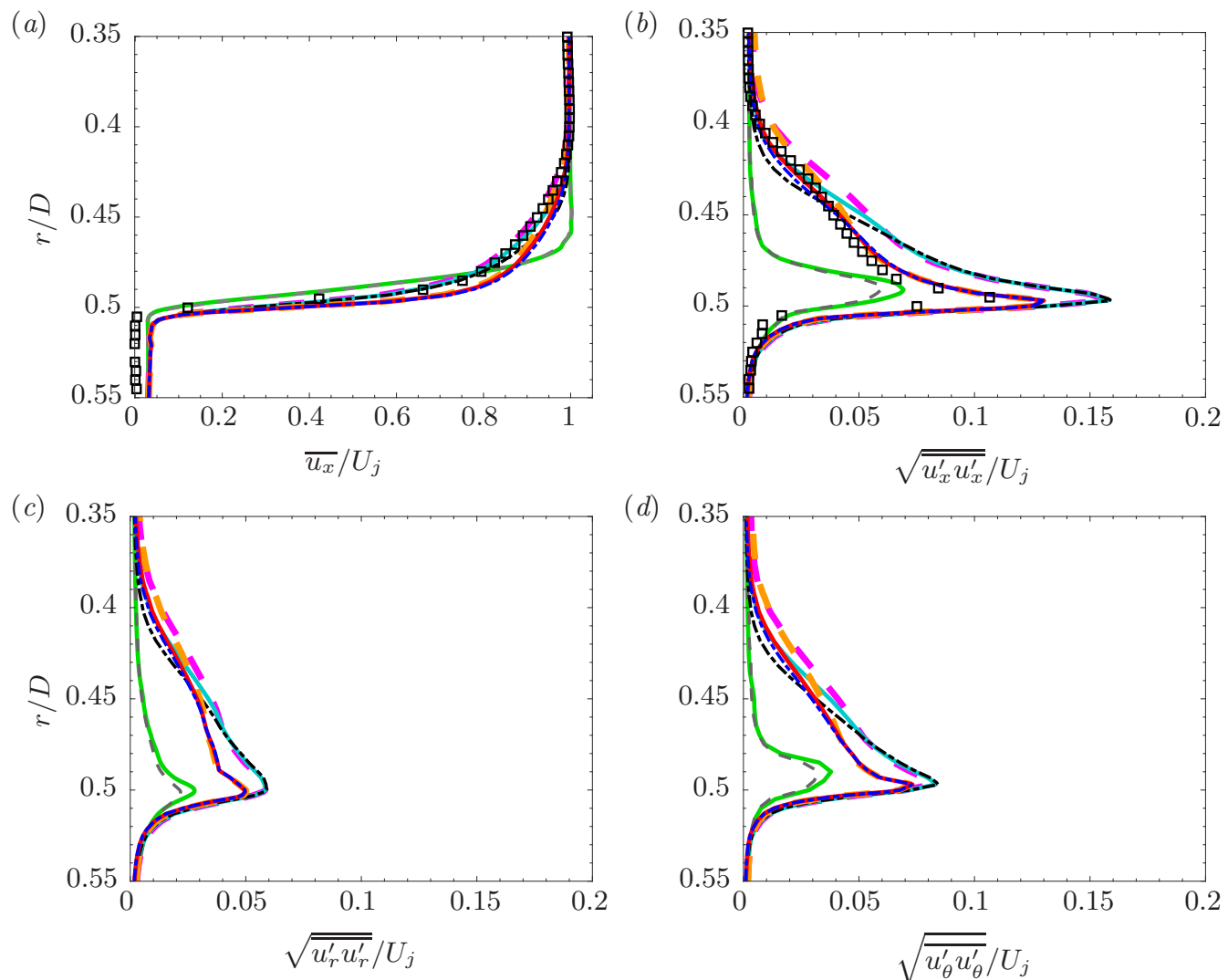

Figure 6. (Colour online) Nozzle-exit boundary layer profiles at $x / D=0.04$ of $(a)$ the mean streamwise velocity and $(b-d)$ the RMS values of the fluctuating velocity components: experiment ( $\square$ hot-wire), baseline LES 10M ( - - ) and 64M ( - ), and LES with nozzle-interior turbulence modelling BL16M ( - - ), BL16M_Turb2 ( $-\boldsymbol{- a})$, BL16M_Turb ( BL16M_WM $(-\cdot-)$, BL16M_WM_Turb2 $(-1-1)$ and BL16M_WM_Turb $\left(-\frac{}{-}\right)$.

a significant impact for the present configuration. All the simulations with isotropic nearwall grid refinement display small-scale three-dimensional turbulent structures in the boundary layer. Depending on the addition of synthetic turbulence and/or wall modelling, the development of turbulence near the walls and in the nozzle core flow differs. Without synthetic turbulence, the internal boundary layer undergoes transition over a long stretch of the nozzle for the case BL16M, and more uniformly around $x / D=-2$ for the case BL16M_WM with wall modelling. With synthetic turbulence, more fluctuations are visible in the pressure and temperature field in the vicinity of the trip, in particular for the cases with the high amplitude coefficient (i.e., suffix Turb2). However, the flow field within the last one diameter before the nozzle exit looks qualitatively similar in all cases with nozzle-interior turbulence modelling.

\subsubsection{Nozzle-exit velocity statistics}

Nozzle-exit profiles of velocity statistics are plotted in figure 6 . Both experimental hotwire measurements and LES results are reported at the same location just downstream of the nozzle exit, at $x / D=0.04$. The slight mismatch in mean velocity for $r / D>0.5$ is caused by the small coflow $M_{\infty}=0.009$ imposed in the simulation.

For both baseline cases, the mean (time-averaged) streamwise velocity profiles are identical and correspond to the typical laminar profile. The turbulence intensities in 
figure $6(b-d)$ all show similar characteristics, with a single wider peak and lower RMS values. In contrast, the nozzle-exit boundary layer in the experiment is turbulent, thanks to the azimuthally homogeneous carborundum strip upstream in the pipe. The RMS peaks are therefore largely under-predicted and the boundary layer is too thin for both LES $10 \mathrm{M}$ and 64M.

With isotropic near-wall grid refinement, all the nozzle-exit boundary layers now exhibit turbulent mean and RMS velocity profiles, with larger fluctuation levels near the wall. Much like the nozzle-exit boundary layer measurements of Fontaine et al. (2015), the present turbulence intensity profiles feature two distinct regions. The first region, which Fontaine et al. (2015) refer to as the "boundary layer remnant", is characterized by a relatively shallow rise, up to $r / D \approx 0.47$ in our study. This region is here present in both experiment and simulations, and, for the simulation, is sensitive to the amplitudes of synthetic turbulence and/or presence of wall modelling. The second region, which they associate with the inflectional instability of the free shear profile, is characterized by a sharp peak in RMS levels near $r / D \approx 0.5$. In that region, the LES results collapse onto two distinct curves, depending on whether or not wall modelling is used. While the nozzle-exit RMS levels are over-predicted compared to experiment for cases BL16M, BL16M_Turb and BL16M_Turb2 (see Figure 6(b)), the cases with wall modelling show less overshoot and better agreement. Here, the effect of the wall model is significant and beneficial: the most important region in terms of the initial growth rate of wavepackets is this "shear-layer" region, where the correct RMS underpins the correct velocity gradient. Over-prediction of near-wall fluctuations is a characteristic feature of under-resolved LES. Even the present choice of 20 points across the nozzle-exit boundary layer thickness is coarse in terms of viscous units at the wall. Based on the resolution in the first LES cell from the nozzle internal surface, the wall-normal grid spacing in wall units $y_{L E S}^{+}$is in the 130 to 175 range, and about 200 to 240 for the streamwise and azimuthal grid spacing, depending on the case and streamwise location. In a corresponding DNS, the typical values would be about 1 in the normal direction, 10 to 20 in the streamwise direction and 5 to 10 in the azimuthal direction. Therefore, the turbulent boundary layer needs to be in the wallmodelled LES regime. The physics in the viscous sublayer are now modelled with the $1 \mathrm{D}$ RANS, leading to an average $y_{R A N S}^{+} \approx 0.7$ for the first RANS cell.

Finally, the addition of synthetic turbulence has less impact than mesh refinement and wall modelling. Two different levels of amplitudes for the synthetic turbulence were tested (see table 1), and the change in fluctuation amplitude can clearly be seen in figure 5 at the location of the trip, for instance in cases BL16M_Turb and BL16M_Turb2. However, as more realistic turbulence develops, the differences in flow structures at the wall only persist for about $0.5 D$ downstream of the trip, and visually similar turbulent boundary layers are then observed beyond that point. As shown in figure 6, the nozzle-exit boundary layer profiles in the "shear-layer" region are essentially independent of the initial choice (or absence) of synthetic fluctuations. The main discernable differences are observed in the "boundary layer remnant", where the turbulence levels in the nozzle core flow away from the walls are slightly larger with the high amplitude synthetic turbulence.

\subsubsection{Centerline and lipline profiles}

The streamwise velocity statistics along the centerline and lipline (i.e., $r / D=0.5$ ) in figure 7 also show improved results for the LES cases with nozzle-interior turbulence modelling. The most drastic change can be observed in the fluctuation amplitude along the lipline in figure $7(d)$ where the fluctuation overshoot around $x=0.5 D$ (related to the 
14

$(a)$

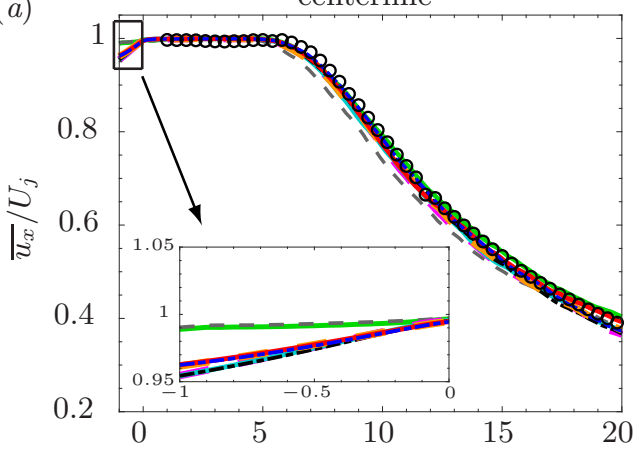

(c) 0.1

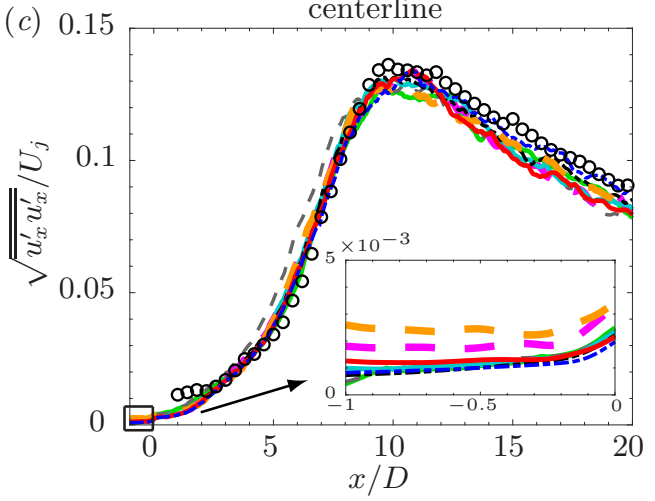

G. A. Brès et al.

(b)

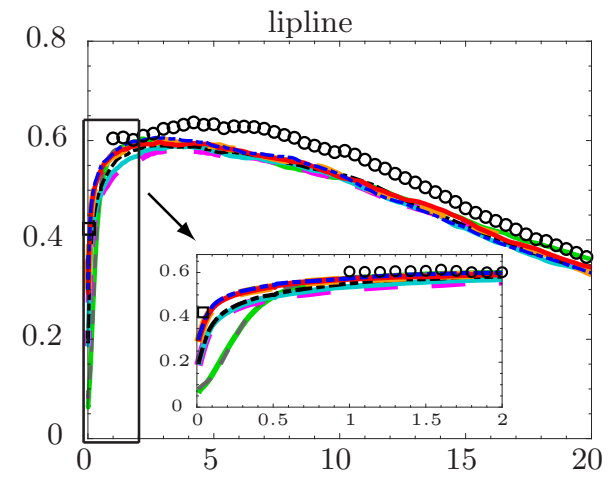

(d)

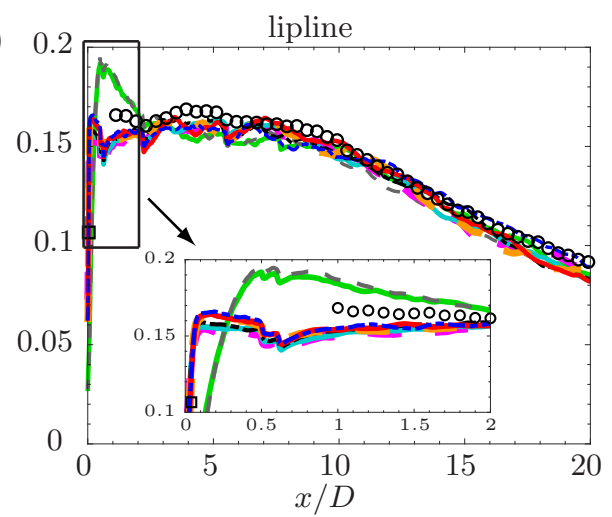

Figure 7. (Colour online) Profiles along the centerline and lipline of $(a, b)$ mean and $(c, d)$ RMS streamwise velocity: experiment ( $\square$ hot-wire, o PIV), baseline LES 10M ( --- ) and 64M ( - ), and LES with nozzle-interior turbulence modelling BL16M ( - - $)$, BL16M_Turb2

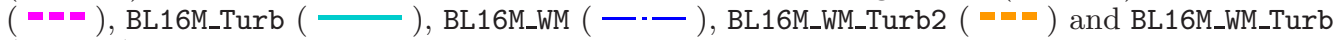
$(-)$.

shear-layer laminar-to-turbulent transition) is present in both baseline LES, independent of the resolution in the jet plume, but is nearly removed with improved treatment of the internal nozzle dynamics.

For the centerline profiles, the main feature is the under-prediction of the length of the potential core $x_{c}$ (defined as the distance up to which the streamwise velocity is greater than $95 \%$ of the jet exit velocity) for the baseline case 10M. The early termination of the potential core results in the shift of the peak RMS levels further upstream. As expected, the grid refinement in the jet plume for case 64M slightly improves the prediction of turbulent mixing, and of $x_{c}$, but the RMS levels remains well under-predicted. Better improvements are actually obtained on the standard mesh for all the cases with nozzleinterior turbulence modelling. Inside the nozzle, all the simulations show very low nozzle core-turbulence levels (see insert in figure $7(c)$ ). As discussed in the previous section, slightly larger values are observed for the two cases with high initial amplitude of the synthetic turbulence (i.e., suffix Turb2)

Overall, the wall-modelled LES cases provide arguably the best match with the PIV measurements, in particular in the very-near nozzle region $x / D<0.5$. Due to the relatively short simulation time used for these preliminary comparisons, the statistics shows some variations between the different cases, in particular for $x / D>8$, in the fullydeveloped mixing jet region downstream of the potential core, where the statistics are more significantly underpinned by low-frequencies, difficult to converge with the short 

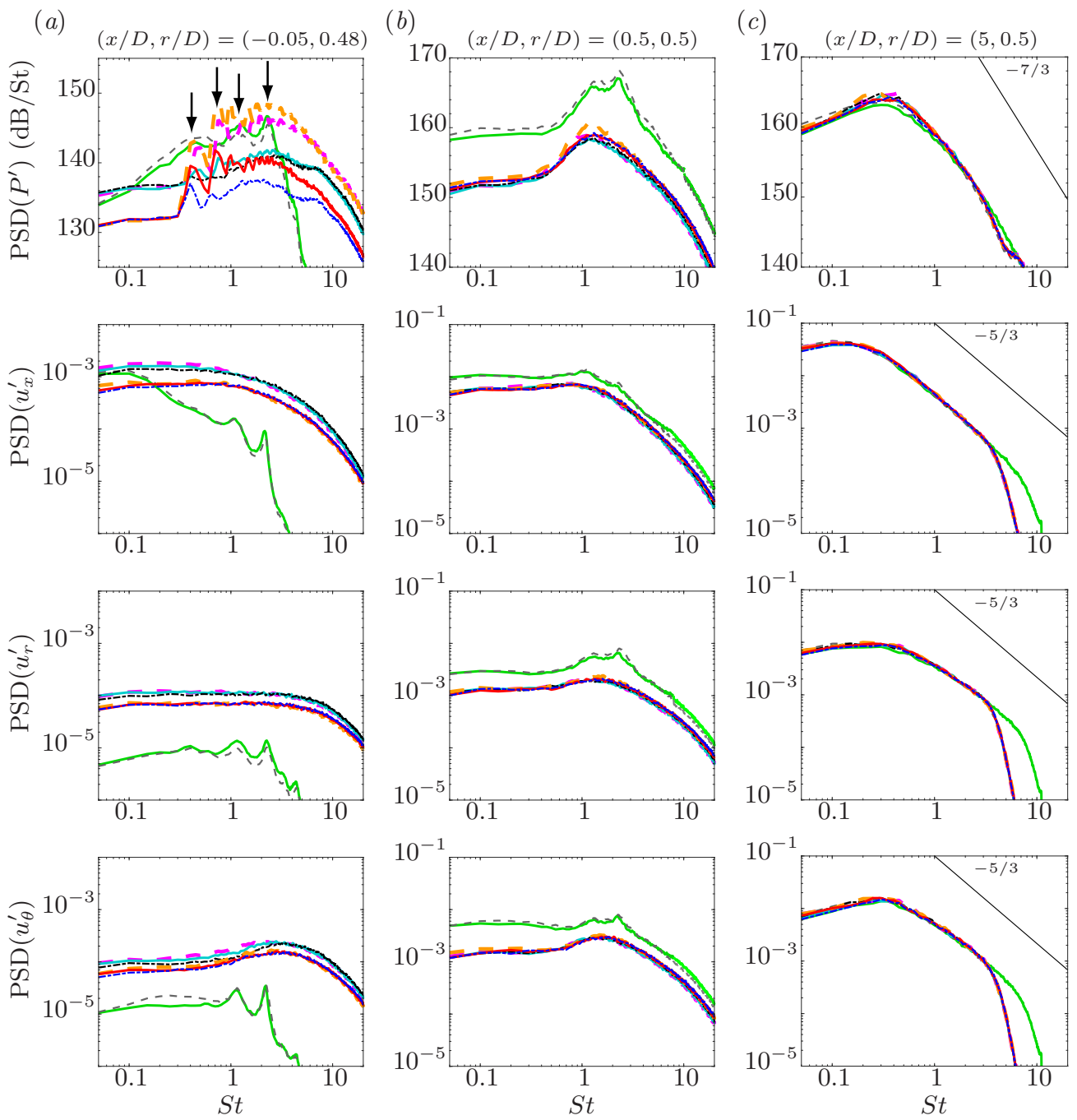

Figure 8. (Colour online) Spectra of pressure and velocity fluctuations along the lipline $(a)$ at $(x / D, r / D)=(-0.05,0.48),(b)(0.5,0.5)$ and $(c)(5,0.5)$ : baseline LES 10M $(---)$ and 64M ( - ), and LES with nozzle-interior turbulence modelling BL16M ( - - ), BL16M_Turb2 $(-\mathbf{m})$, BL16M_Turb $(-)$, BL16M_WM $(-\cdots)$, BL16M_WM_Turb2 $(-m)$ and BL16M_WM_Turb ( - ). The arrows indicate the frequencies of the trapped acoustic waves (see Appendix C).

simulation time. Higher-order moments such as RMS and skewness are, of course, more sensitive to statistical convergence and spatial resolution. Specifically, some of the sharp changes in slope in the RMS profiles are related to transitions in mesh resolution, as corroborated by the mesh spacing curves in figure 3 . These features are discussed in greater details in $\S 4.1$ for the LES with extended simulation time and additional refinement in the jet plume.

\subsubsection{Pressure and velocity fluctuation spectra}

Figure 8 shows the power spectral density (PSD) of the pressure fluctuations (in $\mathrm{dB} / \mathrm{St}$ ) and of the three components of the velocity fluctuations in cylindrical coordinates (nondimensionalized by $U_{j}^{2} / S t$ ) as a function of frequency in Strouhal $S t=f D / U_{j}$. The spectra are directly computed from the flow-field time histories recorded along the lipline 
at $(x / D, r / D)=(-0.05,0.48),(0.5,0.5)$ and $(5,0.5)$ for 36 equally-spaced locations in the azimuthal direction. Because of the azimuthal symmetry of the geometry, these locations are statistically equivalent and the resulting spectra are azimuthally averaged.

The first position $(x / D, r / D)=(-0.05,0.48)$ is representative of the near-wall flow inside the nozzle. As expected, for the baseline cases $10 \mathrm{M}$ and $64 \mathrm{M}$ with initially laminar boundary layers, the velocity fluctuations have much lower levels and no discernible highfrequency content. In contrast, all the simulations with nozzle-interior flow modelling display turbulent spectra with broadband frequency content. For these cases, the velocity spectra collapse onto two curves of similar shape but different amplitude, depending on whether wall modelling is applied or not. As mentioned in the previous section, overprediction of near-wall fluctuations is a characteristic feature of under-resolved LES and all the simulations without wall modelling exhibit higher levels of velocity fluctuations. At this location, the velocity spectra are independent of the initial choice (or absence) of synthetic fluctuations, much like the nozzle-exit velocity profiles in the "shear-layer" region discussed in $\S 3.1 .2$. In contrast, the pressure spectra show some sensitivity to the synthetic turbulence parameters. Namely, higher initial amplitude of the synthetic turbulence inside the nozzle leads to higher levels of pressure fluctuations in the midto high-frequency range. Here, another interesting feature is the presence of tones in the pressure spectra at specific frequencies for all the simulations. Some of the tones are also visible in the velocity spectra of the baseline cases $10 \mathrm{M}$ and $64 \mathrm{M}$ because of the low fluctuation levels. These discrete tones are characteristics of a novel class of resonant acoustic waves which are trapped within the potential core of the jets and transmit some of the energy into the nozzle (see Appendix C).

The second position $(x / D, r / D)=(0.5,0.5)$ corresponds to the location of peak RMS overshoot along the lipline related to the shear-layer laminar-to-turbulent transition in the baseline LES. Therefore, higher fluctuation levels are observed for both cases 10M and $64 \mathrm{M}$ with initially laminar jet, compared to all the other cases with initially turbulent jet, in particular in the pressure spectra. For the simulations with nozzle-interior flow modelling, all the spectra now collapse on a single broadband curve, independently of the use of wall model or presence/initial amplitude of the synthetic turbulence inside the nozzle. Here, some of the tones associated with the trapped acoustic waves are still visible, while others have been overwhelmed by the increased turbulence levels.

The third position $(x / D, r / D)=(5,0.5)$ is located along the lipline towards the end of the potential. As the turbulence continues to develop, the fluctuation levels increase and the spectra shift to lower frequencies, with similar shape and frequency content for all the simulations and variables. In the inertial subrange, all the spectra follow the expected slope of energy cascade in isotropic turbulence, i.e., $-7 / 3$ for pressure and $-5 / 3$ for velocity, up to the grid cut-off frequency $S t \approx 3.5$. The only noticeable exception is the case $64 \mathrm{M}$ with refinement in the jet plume, in which the added grid resolution leads to a higher cut-off frequency around $S t \approx 6.8$

\subsection{Effects of nozzle-interior turbulence modelling on far-field acoustic results}

Figure 9 compares power spectral density (PSD) of pressure fluctuations and overall sound pressure level (OASPL) between experiment and LES cases with and without nozzle-interior turbulence modelling. The PSD is computed with Welch method (block size of $2048,75 \%$ overlap), bin-averaged (bin size $\Delta S t=0.05$ ) and reported in $\mathrm{dB} / S t$, following the same non-dimensionalization as the experiment (see Appendix B). Similar to the direct computation of spectra discussed in $\S 3.1 .4$, the $\mathrm{FW}-\mathrm{H}$ predictions are performed for 36 equally-spaced microphones distributed along the azimuthal angle, and the resulting spectra are azimuthally averaged. The same procedure is applied for calcu- 

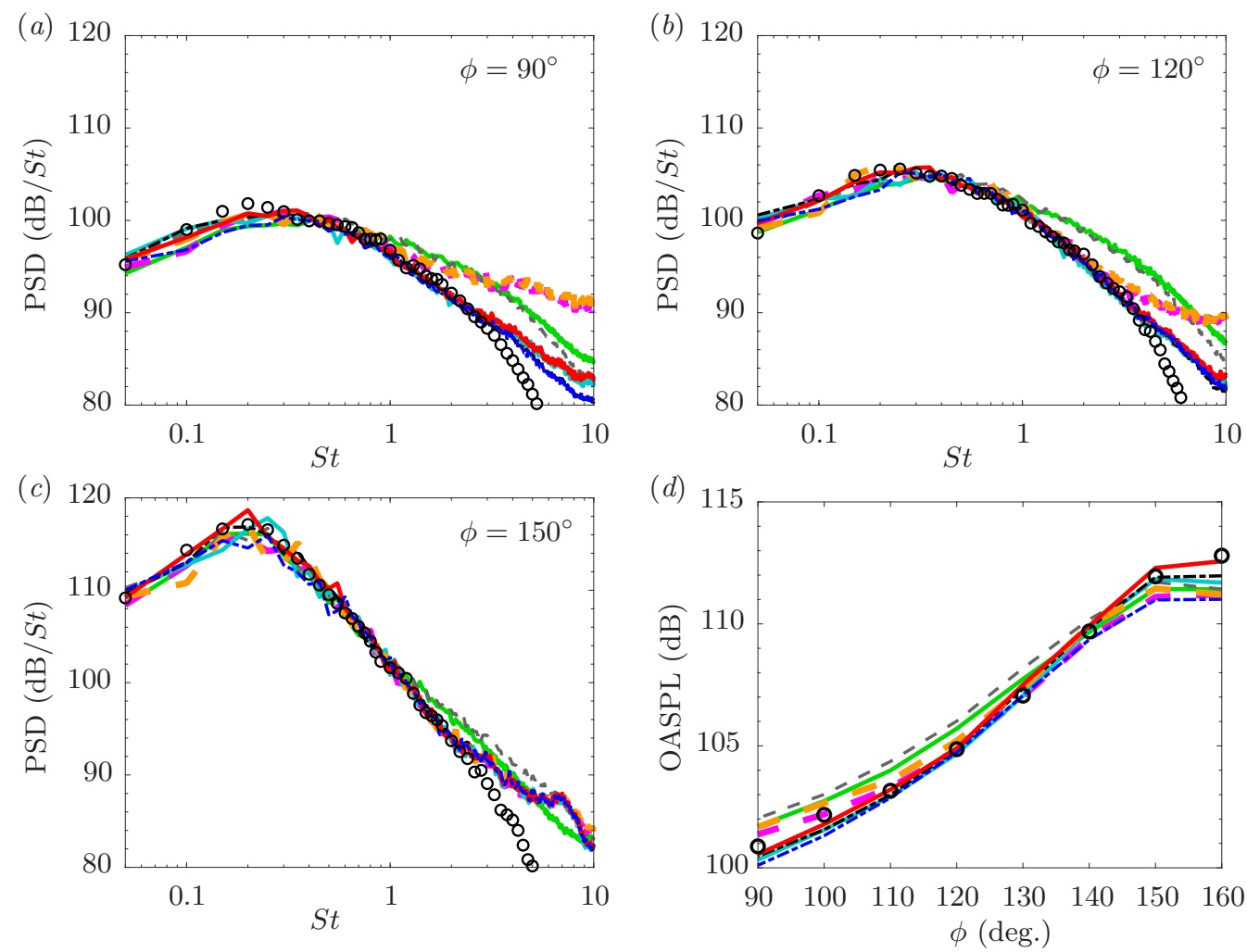

Figure 9. (Colour online) Power spectra density of pressure on the polar microphone array at $50 D$ from the nozzle exit $(a-c)$ at various angles $\phi$ and $(d)$ overall sound pressure levels: experiment ( $\circ$ ), baseline LES 10M ( --- ) and 64M ( _ $)$, and LES with nozzle-interior turbulence modelling BL16M ( - - - ), BL16M_Turb2 ( $-\mathbf{m})$ ), BL16M_Turb (

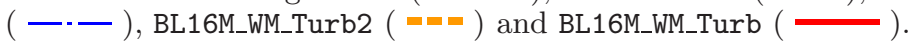

lation of the OASPL in $\mathrm{dB}$, where the frequency range considered for the integration is $0.05 \leqslant S t \leqslant 3$. To evaluate uncertainty in the experimental noise data, basic techniques were used to estimate the errors due to the microphone sensitivity, statistical errors and errors associated with measurement repeatability. The latter was found to be the main source of uncertainty, in general less than $0.5 \mathrm{~dB}$.

For the baseline cases $10 \mathrm{M}$ and $64 \mathrm{M}$, the noise spectra are reasonably well captured up to $S t \approx 1$. For higher frequencies however, the noise levels from these simulations are overpredicted by the same amount for both grids, indicating that refinement in the jet plume will not reduce the discrepancy. This is observed for sideline angles $90^{\circ} \leqslant \phi \leqslant 120^{\circ}$, where the large-scale mixing noise is less dominant. For shallow angles to the jet axis, e.g. $\phi=150^{\circ}$, this high-frequency over-prediction is less severe but the peak radiation around $S t=0.2$ is now under-predicted. These trends translate into discrepancies of approximately 1.5 to $2 \mathrm{~dB}$ in the OASPL, with over-prediction at sideline angles and under-prediction aft.

With nozzle-interior turbulence modelling, the over-prediction observed at high frequencies is eliminated, with the notable exception of the case with high amplitude synthetic turbulence (i.e., suffix Turb2). For these cases, there is an evident change of slope and excess high-frequency noise for $S t>2$ particularly visible at sideline angles, which is likely related to the increase in pressure fluctuations and core-turbulence levels inside the 
nozzle, as previously discussed. The same trends have been reported in the experimental study by Zaman (2012) where larger spectra amplitudes were observed with the application of turbulence-generating grids. In the experiment, the increase was also generally more pronounced at $90^{\circ}$ and higher frequencies.

Aside from these two cases, good agreement with experimental measurements is obtained for the present mesh, which is of modest size, at all angles and frequencies up to $S t \approx 2-3$, consistent with the estimate from the grid design. The resulting OASPL directivity curve in figure $9(d)$ now generally lies within experimental uncertainty, with less than $1 \mathrm{~dB}$ difference for most angles. The discrepancies appears to be mostly due to the variations in low frequencies related to the relatively short simulation time (see statistical convergence and grid resolution study in §4.1). Like the flow field results discussed in the previous section, it was found that the grid adaptation has the most significant impact on far-field noise predictions, while the low amplitude synthetic turbulence and wall model have more subtle effects. With the exception of the two LES cases with high input turbulence, the spectra do not contain discernible tones nor visible numerical artifacts that could be directly related to the added modelling inside the nozzle.

\section{Laminar versus turbulent jets}

4.1. Database validation: statistical convergence and grid resolution study

Based on the results presented above, the turbulent case BL16M_WM_Turb and laminar case $10 \mathrm{M}$ were selected for further analysis and comparisons. The total simulation time in both cases was increased to $t_{\text {sim }} c_{\infty} / D=2000$. Finally, to investigate grid convergence, an additional simulation for the same configuration and numerical setup than BL16M_WM_Turb was performed on the refined mesh, the i.e., 69 million cvs grid with double the resolution in the jet plume (see table 1).

\subsubsection{Jet plume statistics}

Figure 10 shows comparisons of the streamwise velocity statistics in the jet plume between PIV and LES for the extended simulations. The corresponding centerline and lipline profiles are presented in figure 11. As discussed in Appendix A, reliable PIV measurements are not available for $x / D<1$ because of edge effects near the nozzle.

Despite the significant differences in grid resolution in the jet plume, both simulations BL16M_WM_Turb and BL69M_WM_Turb with nozzle-interior turbulence modelling give similar flow-field results, both in good agreement with the experimental measurements. Compared to the profiles in figure 7 with statistics computed over 600 acoustic time units (i.e., the duration from the preliminary study), the predictions for the extended simulations show improvements in the statistical convergence. The mesh refinement in the jet plume for case BL69M_WM_Turb also provided some improvements of the artifacts associated with transitions in mesh resolution. As mentioned in $\S 3.1$, the discontinuities in RMS levels observed at $x / D \approx 0.5,2.1$ and 5 in figures $10(b)$ and $11(b)$ correspond to unstructured grid transitions. With smaller changes in grid spacing on the refined mesh, the grid imprint on RMS levels is reduced in the refined case. For both extended simulations with nozzle-interior turbulence modelling, the length of the potential core $x_{c}$ is well predicted (see table 3). As expected, grid refinement in the jet plume tends to increase the value of $x_{c}$ and shift the centerline peak RMS fluctuations further downstream. Likewise, after the end of the potential core, the refined case tends to display slightly higher mean and 

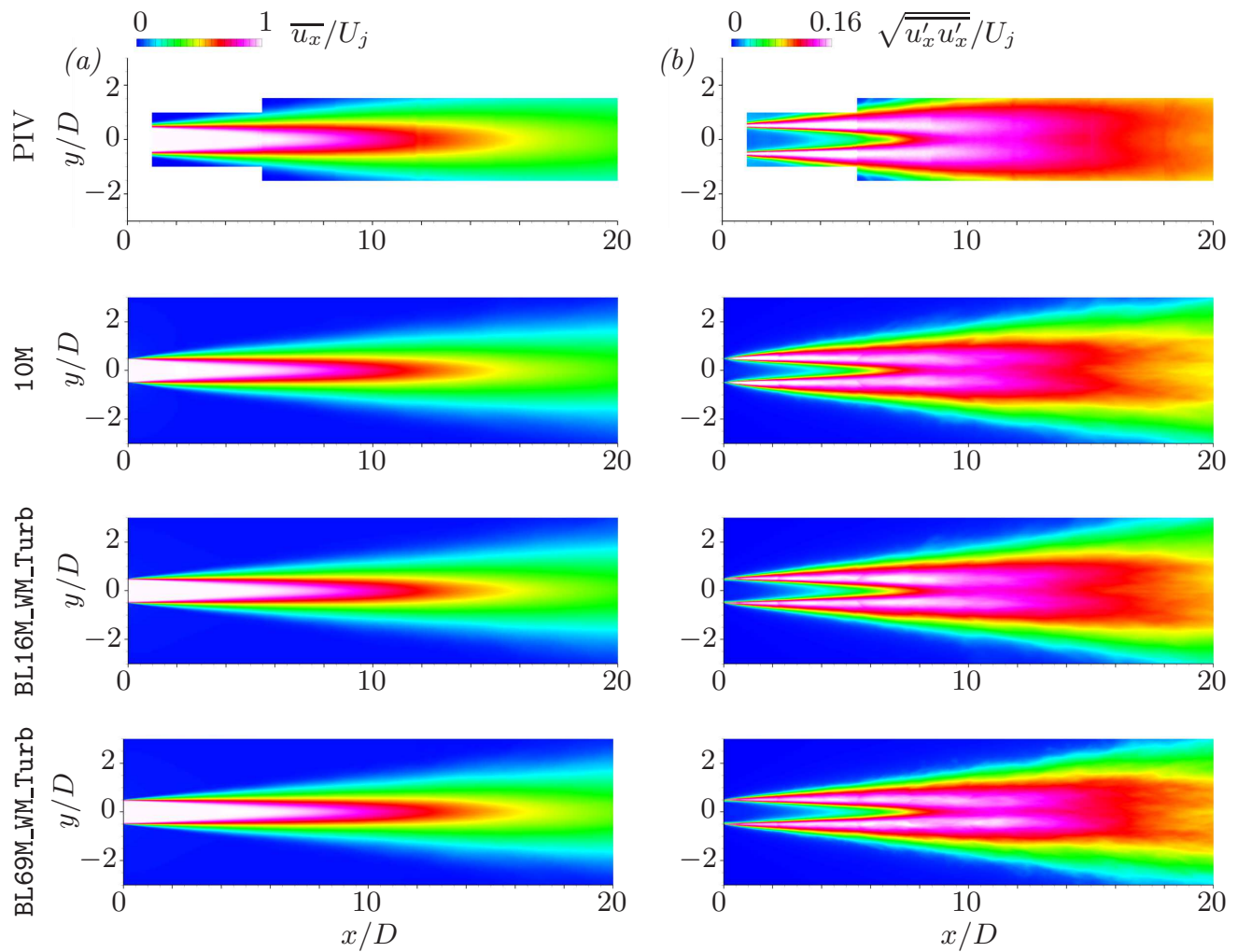

Figure 10. (Colour online) Contours of $(a)$ mean and (b) RMS streamwise velocity in the mid-section plane $(z=0)$ : experimental PIV (top row), extended baseline LES 10M and extended LES with nozzle-interior turbulence modelling BL16M_WM_Turb and BL69M_WM_Turb.
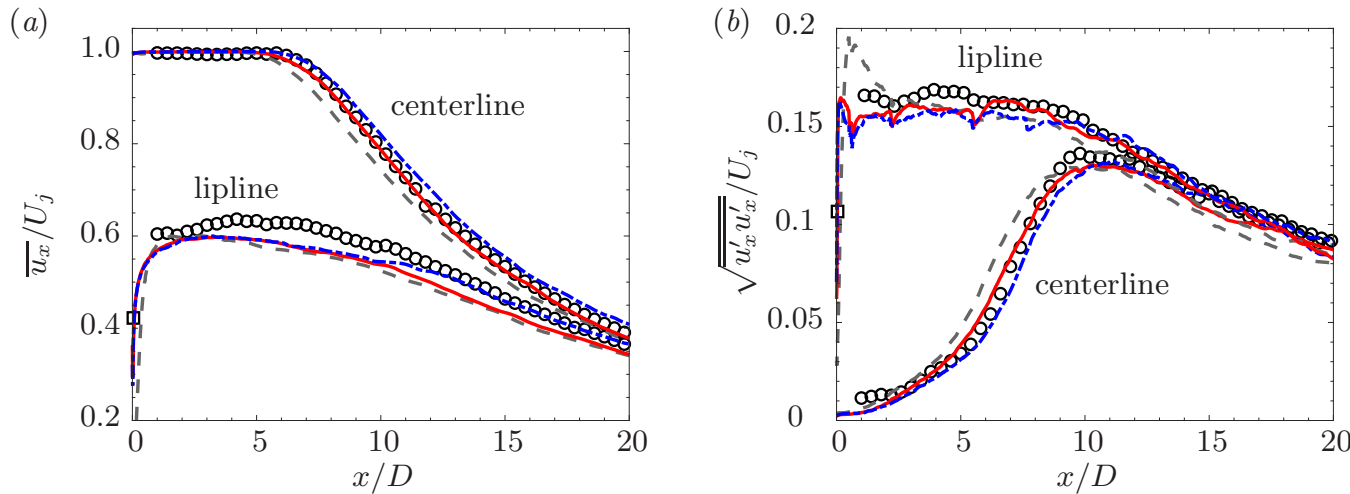

FiguRE 11. (Colour online) Centerline and lipline profiles of ( $a$ ) mean and (b) RMS streamwise velocity: experiment ( $\square$ hot-wire, o PIV), extended baseline LES 10M ( - - - ) and extended LES with nozzle-interior turbulence modelling BL16M_WM_Turb ( — $)$ and BL69M_WM_Turb ( - - - ).

RMS values than the standard case, as the increase in resolution in that region leads to prediction improvements of the turbulent mixing. 


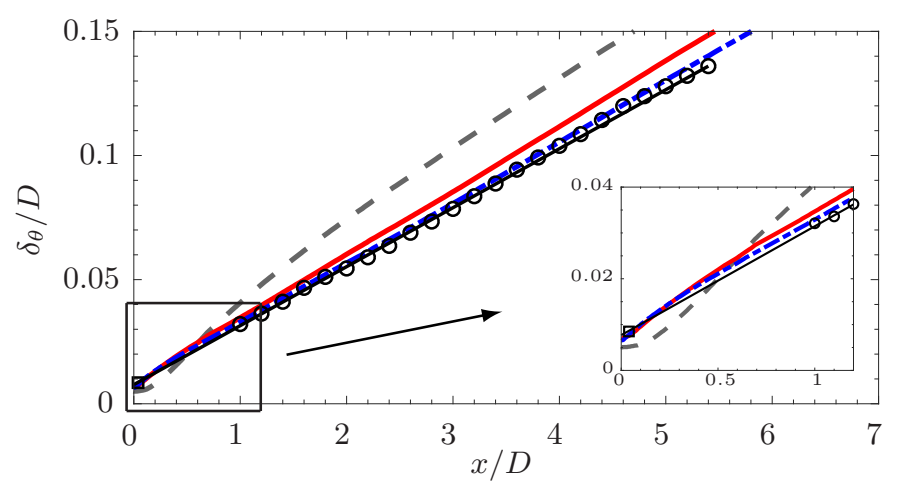

Figure 12. (Colour online) Profiles of the shear-layer momentum thickness $\delta_{\theta}$ : experiment ( $\square$ hot-wire, ○ PIV, — linear interpolation), extended baseline LES 10M ( - - - ) and extended LES with nozzle-interior turbulence modelling BL16M_WM_Turb ( - ) and BL69M_WM_Turb $(-\cdot-)$.

\subsubsection{Nozzle-exit conditions and shear-layer development}

The shear-layer momentum thickness $\delta_{\theta}$ are presented in figure 12. Similarly to Bogey \& Bailly (2010), $\delta_{\theta}$ is estimated as

$$
\delta_{\theta}(x)=\int_{0}^{r_{0.05}} \frac{\overline{u_{x}}(x, r)}{\overline{u_{x}}(x, 0)}\left(1-\frac{\overline{u_{x}}(x, r)}{\overline{u_{x}}(x, 0)}\right) d r,
$$

where $\overline{u_{x}}$ is the time- and azimuthal-averaged streamwise velocity. The integral radial bound $r_{0.05}$ accounts for the slow coflow and corresponds to the distance where $\overline{u_{x}}\left(x, r_{0.05}\right)-U_{\infty}=0.05 \overline{u_{x}}(x, 0)$. The same approach is used to estimate the displacement thickness $\delta^{*}$. Table 3 summarizes all the nozzle-exit boundary layer properties predicted from simulations and estimated from the experimental PIV using linear extrapolation to $x / D=0$. As the shape factor $H=\delta^{*} / \delta_{\theta}$ varies from 2.59 for fully laminar flow to approximately 1.4 for fully turbulent flow (Schlichting \& Gertsen 2000), the results confirm the initially laminar and turbulent state of the jets for the different LES. Here the estimated momentum thickness is also comparable to the values $\delta_{\theta} / D \approx 0.0055$ to 0.0213 reported in the recent experiments by Fontaine et al. (2015) with similar convergent-straight nozzles and operating conditions.

Figures 13 and 14 shows the evolution of the mean streamwise velocity and streamwise turbulence intensity at different axial locations upstream and downstream of the nozzle exit. As previously discussed, both LES cases BL16M_WM_Turb and BL69M_WM_Turb have the same adapted mesh inside the nozzle and the same synthetic turbulence and wall modelling applied to the nozzle internal walls. This leads to identical profiles for $x / D<0$ and similar integral quantities for the nozzle-exit boundary layer. The only noticeable difference is at $x / D=0.04$ in figure $14(a)$ for the maximum RMS levels around $r / D=0.5$, where the additional resolution in the jet plume for the refined case is better suited to resolve the strong velocity gradients and sharp peak of the RMS levels at the lipline. That peak is missed in the measurement because of limited spatial resolution. For both simulations, the linear growth of the shear-layer starts almost immediately at the nozzle exit and closely matches the experimental value in figure 12 .

In contrast, for the initially laminar jet in simulation 10M, the jet flow development is characterized by different features in three distinct regions. Inside the nozzle, the boundary layer is laminar and the jet remains laminar with limited spreading close to the nozzle exit, up to $x / D \approx 0.2$ (see insert in figure 12). This is followed by a rapid growth 

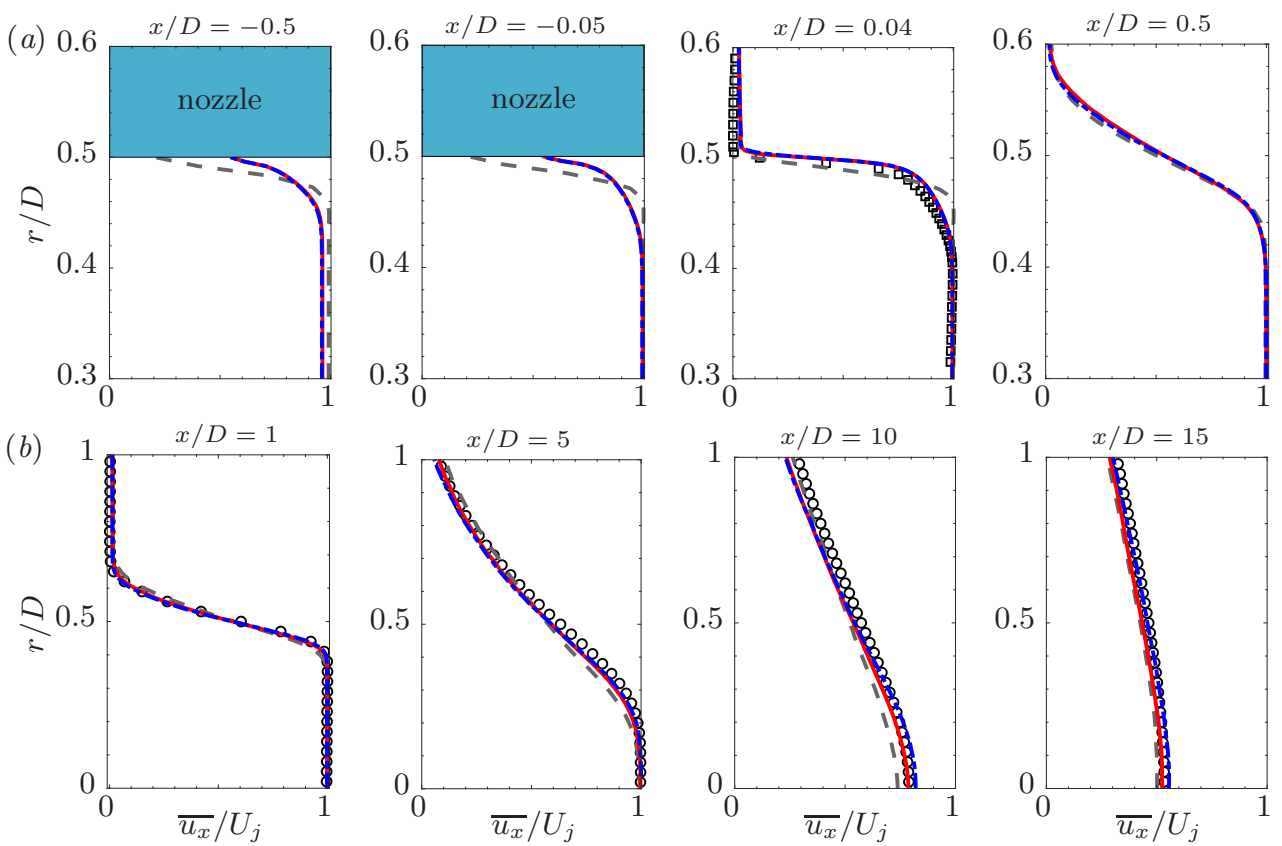

Figure 13. (Colour online) Profiles of the mean streamwise velocity $(a)$ in the near-nozzle region and $(b)$ in the jet plume: experiment ( $\square$ hot-wire, o PIV), extended baseline LES 10M ( -- - ) and extended LES with nozzle-interior turbulence modelling BL16M_WM_Turb ( - ) and BL69M_WM_Turb $(-\cdot-)$.
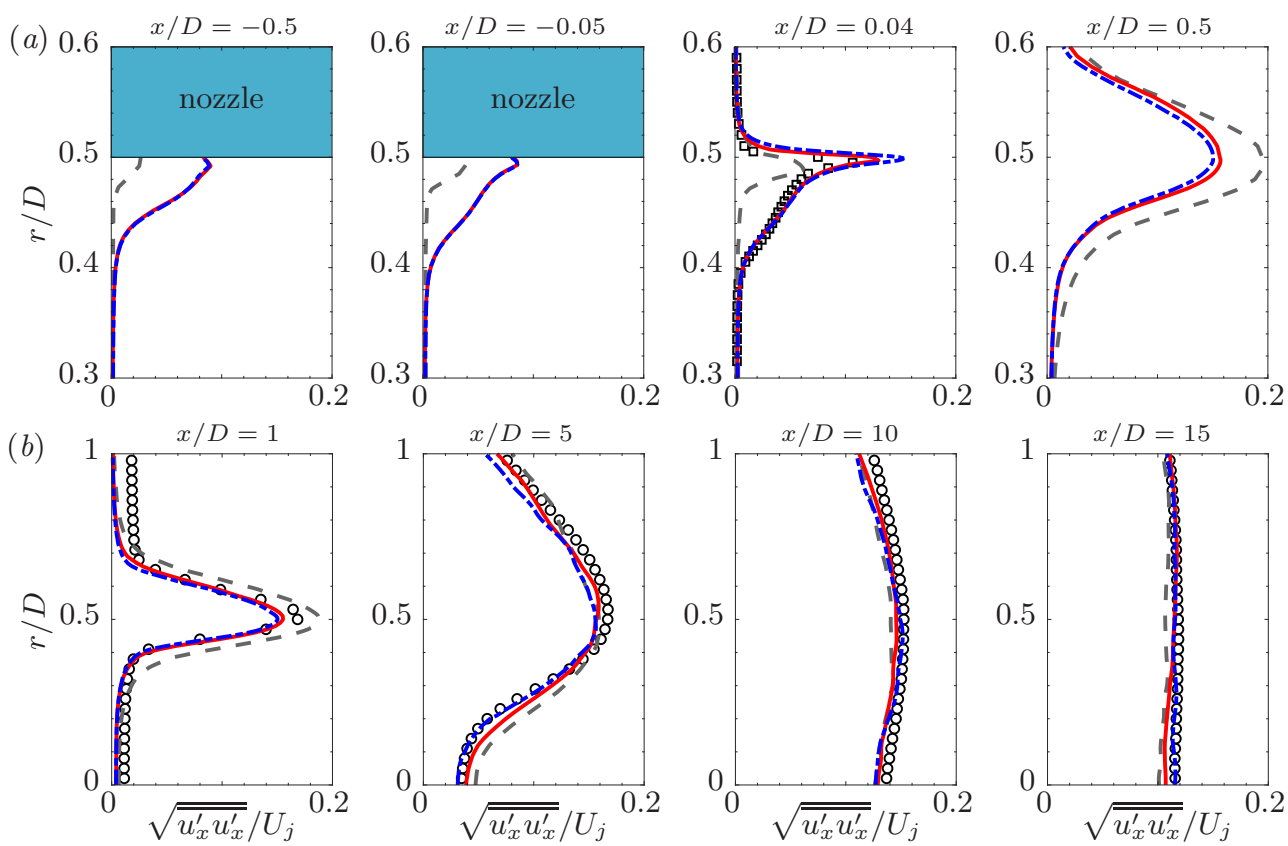

Figure 14. (Colour online) Profiles of the RMS streamwise velocity $(a)$ in the near-nozzle region and $(b)$ in the jet plume: experiment ( $\square$ hot-wire, o PIV), extended baseline LES 10M ( -- $)$ and extended LES with nozzle-interior turbulence modelling BL16M_WM_Turb ( - ) and BL69M_WM_Turb $(-\cdot-)$. 


\begin{tabular}{llccccc}
\hline \multirow{2}{*}{ Approach } & Methodology & $x_{c} / D$ & $\delta_{\theta} / D$ & $\delta^{*} / D$ & $\delta^{99} / D$ & $\mathrm{H}$ \\
Experiment & PIV & 7.5 & 0.0077 & 0.012 & 0.080 & 1.56 \\
Baseline LES & 10M & 6.5 & 0.0051 & 0.013 & 0.039 & 2.54 \\
LES with modelling & BL16M_WM_Turb & 7.3 & 0.0071 & 0.011 & 0.073 & 1.55 \\
& BL69M_WM_Turb & 7.7 & 0.0066 & 0.010 & 0.073 & 1.51
\end{tabular}

TABLE 3. Estimates of the jet potential core length $x_{c}$, nozzle-exit boundary-layer momentum thickness $\delta_{\theta}$, displacement thickness $\delta^{*}$, thickness $\delta^{99}$ and shape factor $H$.

related to the shear-layer laminar-to-turbulent transition, as indicated by the overshoot of the velocity RMS around $x / D=0.5$ in figure 11 and figure $14(a)$. This process then leads to enhanced mixing further downstream, resulting in the larger spreading rate observed in figure 12 and earlier termination of the potential core. Overall, the trends for the potential core length and shear-layer growth are consistent with the results reported by Bogey \& Bailly (2010) for simulations of initially laminar jets at Mach 0.9.

\subsubsection{Far-field acoustics}

In addition to the single microphone in the far field, pressure measurements were also made using a 18-microphone azimuthal ring array whose axial position was varied in order to map the sound field on a cylindrical surface of radius $r / D=14.3$ centered on the jet axis. This microphone ring is also used to perform the azimuthal decomposition of the radiated noise discussed in $\S 4.2$. The complete comparison with the LES predictions is presented in figure 15 for all microphones, with the corresponding overall sound pressure level directivity (OASPL) shown in figure 16.

First, for the initially laminar jet, the results of the extended simulation $10 \mathrm{M}$ confirms the conclusion of the preliminary study: the noise spectra are reasonably well predicted for most angles up to frequency $S t \approx 1$, with over-prediction at higher frequencies and slight under-prediction of the peak radiation around $S t=0.2$. The discrepancies are more pronounced on the cylindrical microphone array (see zoomed-in view of the spectra in figure 18) and lead to the mismatch in shape for the noise directivity observed in the OASPL levels in figure 16. Experimental studies by Brown \& Bridges (2006), Zaman (2012), and Karon \& Ahuja (2013) all reported similar increased levels at high frequencies for subsonic jets with (nominally) laminar initial shear layers, compared to jets with (nominally) turbulent ones. In particular, Brown \& Bridges (2006) applied a thin wrap of reticulated foam metal (RFM) inside their nozzle to trip the boundary layer, similar to the carborundum strip used in the present experiments. The RFM inserts changed the characteristics of the nozzle-exit boundary layer from laminar to turbulent and eliminated the high frequency noise.

For the initially turbulent jets, there is little variation between the results from the standard and refined simulations, for most angles and relevant frequencies. With the extended simulation time, the low frequency part of the spectra shows better convergence compared to the preliminary results in figure 9 and the predictions are further improved, now typically within $0.5 \mathrm{~dB}$ of the measurements. The main discernible differences between the spectra from the two LES are observed in the grid cut-off frequency for the high angles $\phi \geqslant 150^{\circ}$ : at these angles, the limit frequency is about $S t \approx 2$ for the standard case BL16M_WM_Turb and $S t \approx 4$ for the refined case BL69M_WM_Turb with double the resolution in the jet plume. Here, it is important to note that these discrepancies are outside of the main frequency range of interest and with levels 25 to $30 \mathrm{~dB}$ lower that the peak radiated noise, such that they do not significantly impact the predictive 

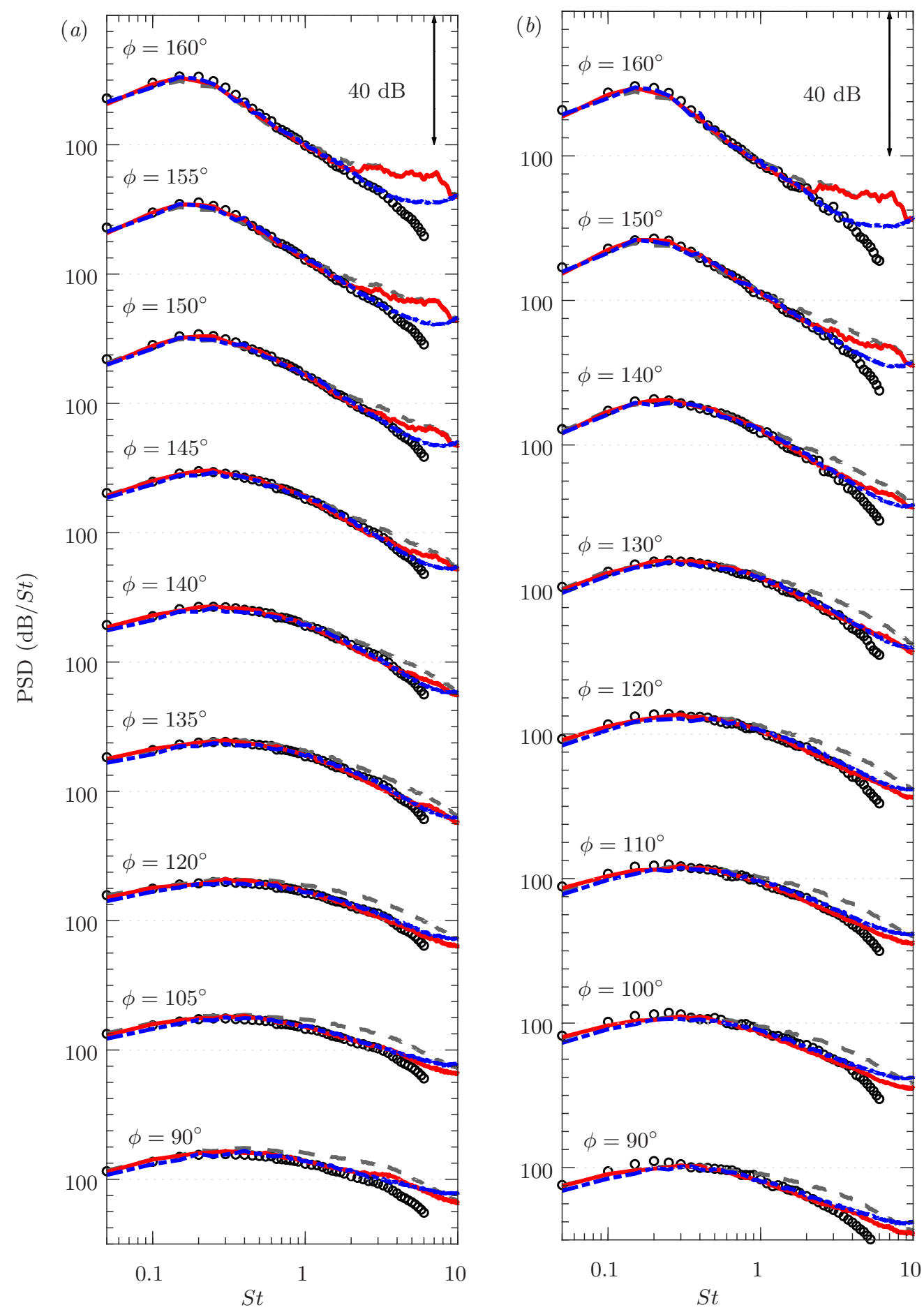

Figure 15. (Colour online) Power spectra density of pressure $(a)$ on the cylindrical microphone array of radius $r=14.3 \mathrm{D}$ and $(b)$ on the polar microphone array at $50 D$ from the nozzle exit for the different angles $\phi$ : experiment ( $\circ)$, extended baseline LES 10M ( --- ) and extended LES with nozzle-interior turbulence modelling BL16M_WM_Turb ( - ) and BL69M_WM_Turb ( - - - ). 

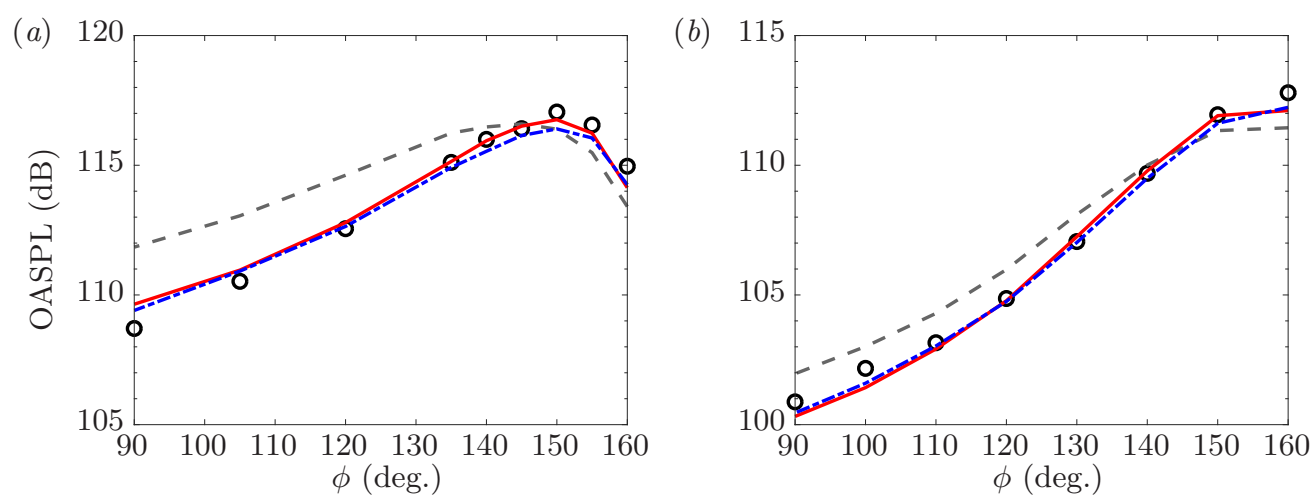

FIGURE 16. (Colour online) Overall sound pressure level directivity $(a)$ on the cylindrical microphone array of radius $r=14.3 \mathrm{D}$ and $(b)$ on the polar microphone array at $50 \mathrm{D}$ from the nozzle exit: experiment ( $\circ)$, extended baseline LES 10M (--- ) and extended LES with nozzle-interior turbulence modelling BL16M_WM_Turb ( — $)$ and BL69M_WM_Turb ( - - - ) .

capabilities nor use of the database for sound-source modelling. Overall, the statistical convergence and grid resolution studies provide thorough validation and confidence in the LES database of case BL16M_WM_Turb, for flow and noise data up to $S t \approx 2-3$. All of the remaining analysis is therefore conducted using that longer database.

\subsubsection{Near-field acoustics}

For the eduction of wavepacket signatures and further investigation of the tones observed in the LES spectra inside the nozzle, the experiment was also instrumented with a 48-microphone cage array consisting in 6 azimuthally equispaced microphones at 7 different locations in the near field on the jet. Figure 17 shows the comparison with the LES predictions at three representative locations: $(x / D, r / D)=(0.12,0.72),(b)(2.00,0.91)$ and $(c)(4.47,1.33)$.

For the microphone ring closest to the nozzle exit, corresponding to a jet inlet angle of $\phi \approx 99.5^{\circ}$, the discrete tones associated with the resonant acoustic waves are again observed in the spectra, consistent with the results inside the nozzle discussed in section §3.1.4. For both simulations with initially turbulent jet, the shape of the spectra and the frequency and amplitude of the tones closely match the experimental measurements. For the simulation with initially laminar jet, the tones are still present at the same frequencies but the overall levels are higher because of the enhanced noise radiation related to the shear-layer laminar-to-turbulent transition. Additional analysis of the discrete tones is presented in Appendix C.

For the near-field microphones further downstream, the same conclusions hold in terms of agreement with experiment for the simulations with initially turbulent jet and overpredictions for the simulation with initially laminar jet. At these locations corresponding to the peak radiation angles (i.e., $\phi \approx 153.9^{\circ}$ and $163.4^{\circ}$ ), the spectra levels are much higher and there is no visible tonal component. As discussed in detail in the work of Towne et al. (2017) and Schmidt et al. (2017), the resonant acoustic waves are trapped within the potential core of the jet and decay rapidly away from the jet. Therefore, there are no discernible tones in figure $17(b)$ and $(c)$, nor in figure 15 for the far-field noise predictions.

\subsection{Azimuthal mode decomposition of the radiated noise}

Dating back to Michalke \& Fuchs (1975), who first argued that low-order azimuthal modes would be the dominant sources of sound in subsonic circular jets, many experi- 
(a)

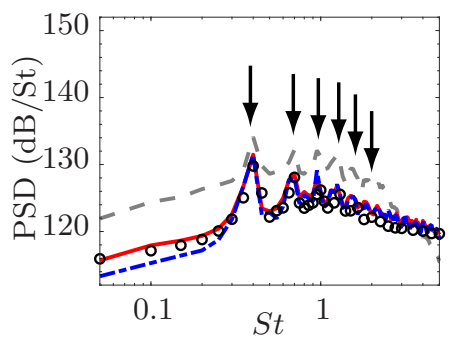

(b)

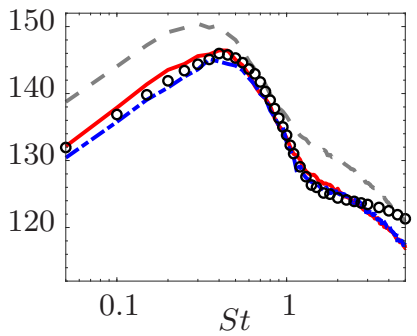

(c)

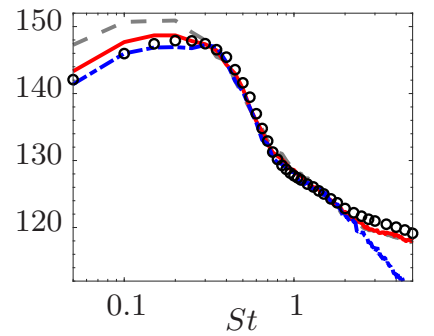

Figure 17. (Colour online) Power spectra density of pressure on the near-field cage microphone array at $(a)(x / D, r / D)=(0.12,0.72),(b)(2.00,0.91)$ and $(c)(4.47,1.33)$ : experiment (o ), extended baseline LES 10M (--- ) and extended LES with nozzle-interior turbulence modelling BL16M_WM_Turb ( -$)$ and BL69M_WM_Turb ( - - $)$. The arrows indicate the frequencies of the trapped acoustic waves (see Appendix C).

mental jet studies have suggested that low-frequency noise (i.e., Strouhal number $S t<1$ ) may be decomposed into just 3 Fourier azimuthal mode $m=0,1$ and 2 (Juvé et al. 1979; Kopiev et al. 2010; Cavalieri et al. 2011, 2012, amongst others). The azimuthal mode analysis is applied to the present experimental and LES databases and extended to higher frequencies, to further investigate the differences observed in radiated noise between jets with laminar and turbulent nozzle-exit boundary layer state. For both experiment and simulation, the azimuthal decomposition is performed using the data from 18 microphones evenly-spaced in the azimuthal direction, on the cylindrical array of radius $14.3 D$, following the procedure described by Cavalieri et al. (2012). The output is the complex acoustic pressure as a function of frequency and azimuthal mode $m$ at each jet inlet angle on the array. The procedure was reproduced using the LES data from 128 evenly-spaced microphones instead of 18 , and provided similar results and conclusions for the azimuthal modes and frequency range considered.

Figure 18 shows the experimental and numerical spectra of the total signal and the first five azimuthal modes for a few representative jet inlet angles $\phi$. In addition, PSD levels from the different modes at selected frequencies are plotted as a function of $\phi$ in figure 19. In these figures, the total noise spectra from experiment (black solid circle), LES cases 10M (dashed grey line) and BL16M_WM_Turb (solid red line) is the same data reported in figure $15(a)$.

For the initially turbulent jet, the agreement between measurement and LES is again excellent, particularly for the first four modes. Figure 19(a-c) shows the PSD values from figure 18 extracted at specific low frequencies $S t=0.1,0.2$ and 0.3 . In the low frequency range $0.05 \leqslant S t \leqslant 0.4$, the axisymmetric azimuthal mode $m=0$ is dominant at the peak radiation angles $\phi=140^{\circ}-160^{\circ}$, followed by mode $m=1$ and then $m=2$. At the lower inlet angles $\phi \leqslant 135^{\circ}$, the mode order (in terms of importance) tends to be reversed, with mode $m=1$ and 2 more energetic than $m=0$, and the differences are less pronounced. Furthermore, the higher-order modes $m \geqslant 3$ have much lower contributions. These results are confirmed by the OASPL curves computed over the full frequency range $0.05 \leqslant S t \leqslant 3$ in figures $20(a)$ and $(b)$. In these figures, the total OASPL is compared to that calculated with selected azimuthal modes retained for the pressure, namely either mode $m=0$ only, modes $m=0$ to 1 , etc $\ldots$ up to modes $m=0$ to 4 . At $\phi=160^{\circ}$, mode $\mathrm{m}=0$ contributes to $\mathrm{OASPL}_{m} / \mathrm{OASPL}_{\text {total }}=86 \%$ of the total acoustic energy, and this value goes to more than $99.2 \%$ when the first 3 modes are considered. Over all angles, the first 3 Fourier azimuthal modes of the LES data recover more than $65 \%$ of the total acoustic energy, which means that a prediction based on these 3 dominant modes would 

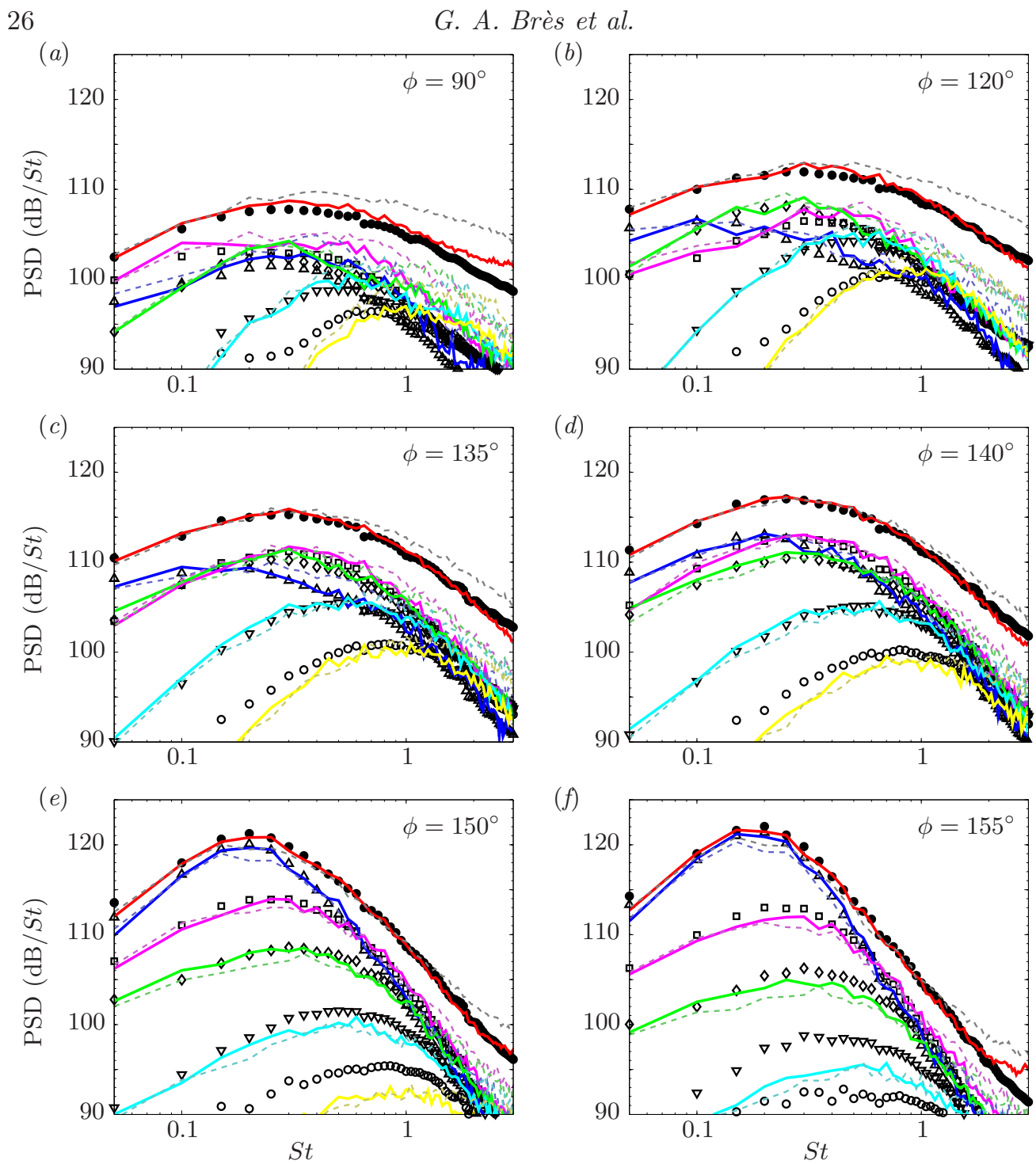

Figure 18. (Colour online) Azimuthal mode decomposition of the radiated noise at specific angles $\phi$ for the experimental data (symbols), initially laminar jet 10M (dashed lines) and turbulent jet BL16M_WM_Turb (solid lines): ( $\bullet,-$ ) total (i.e., all modes); $(\triangle,-)$ mode $m=0$;

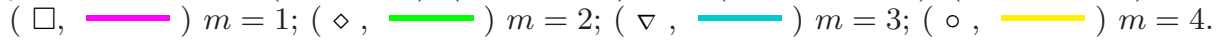

be within $1.9 \mathrm{~dB}$ of the total OASPL value. These results are all consistent with the experimental trend previously reported in the literature. At higher frequencies, the PSD levels are lower and more modes have comparable contributions to the radiated sound (see figure $19(d-f)$ ).

For the initially laminar jet, the same conclusions hold, despite the significant differences in noise levels previously discussed. In the low frequency range $S t<1$ where the radiation from the laminar and turbulent jets are similar, the azimuthal mode decomposition for the LES case 10M provides results similar to those of the turbulent jet. In the higher frequency range, the low azimuthal modes have elevated levels compared to the turbulent case (see figure 19(e-f)). However, these discrepancies appear to be directly 

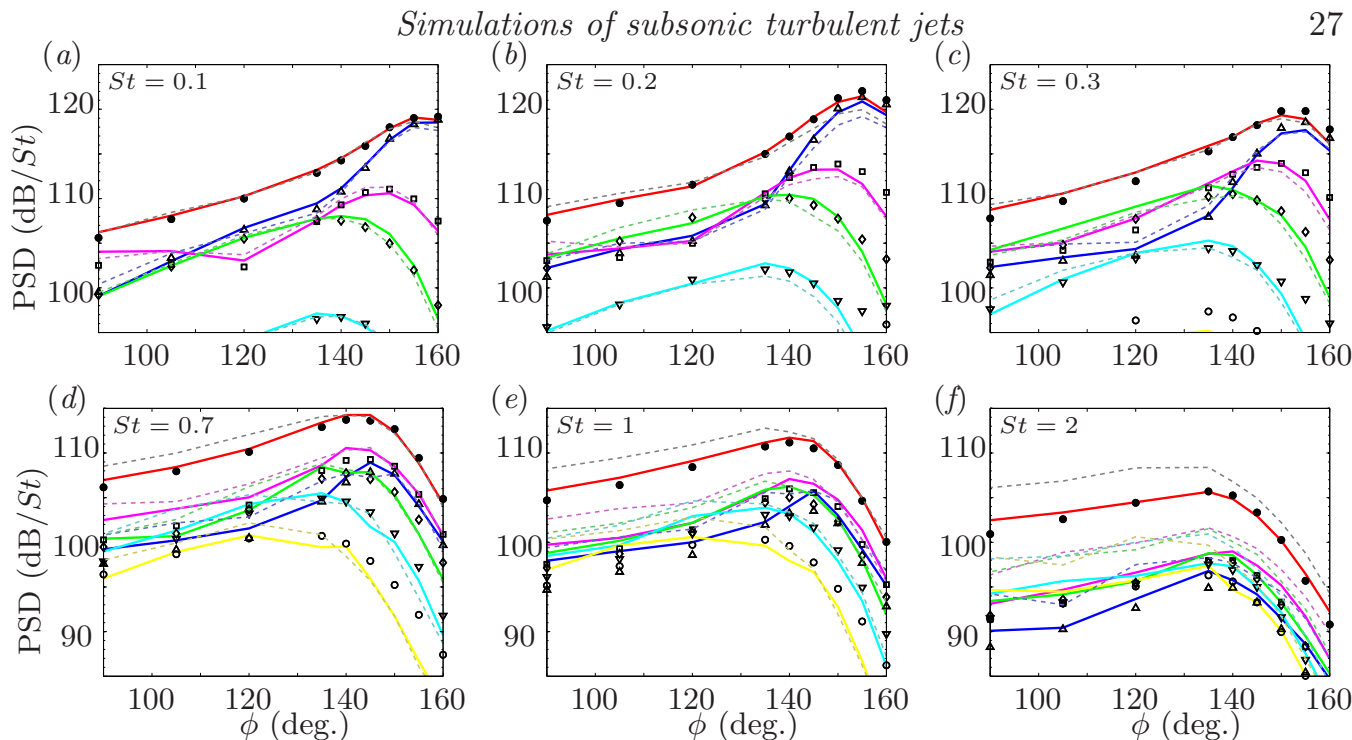

Figure 19. (Colour online) Azimuthal mode decomposition for the radiated noise at specific frequencies $S t$ for the experimental data (symbols), initially laminar jet 10M (dashed lines) and turbulent jet BL16M_WM_Turb (solid lines): ( • , - ) total (i.e., all modes); $(\triangle,-$ - $)$

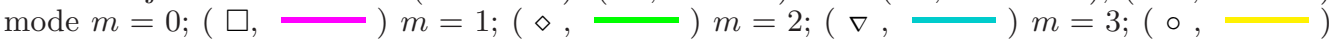
$m=4$.

related to the increase in total noise, while the relative strength and contribution of the different modes are not altered.

To show this more clearly, a mode-independent correction $\triangle \mathrm{OASPL}=\mathrm{OASPL}_{\text {total }}^{\text {turbulent }}$ $\mathrm{OASPL}_{\text {total }}^{\text {case }}$ is computed for each jet inlet angle and applied to the noise directivity in figures $20(a)$ and $(c)$. In this latter figure, the frequency range of the OASPL integration is reduced to $1 \leqslant S t \leqslant 3$, to focus the analysis solely on the frequencies with the largest noise discrepancies between the initially laminar and turbulent jet. Note that for both frequency ranges, $\triangle \mathrm{OASPL}=0$ for the turbulent case and the curves are unchanged. For the laminar case, the correction collapses the total OASPL curve (i.e., dashed grey line) onto the turbulent one (i.e., solid red line) and tends to systematically shift all the results towards the predictions of the turbulent jet case BL16M_WM_Turb. In terms of the mode relative OASPL contribution, figures $20(b)$ and $(d)$ show that both laminar and turbulent jets follow again the same trends, and confirm the strong frequency dependence of the mode ranking. As previously mentioned, the mode $m=0$ is no longer dominant at high frequencies and more azimuthal modes are required there to describe the total noise. Specifically, for $1 \leqslant S t \leqslant 3$, the first 5 modes (instead of 3 ) are now needed to recover more than $65 \%(90 \%)$ of the total acoustic energy for all angles (peak radiation angles).

In summary, the azimuthal mode analysis provides two key results. First, it confirms that the first few azimuthal modes are the main sources of sound for both initially laminar and turbulent jets. And second, it suggests that the significant differences in radiated noise observed at high frequencies between the two jets are not due to a specific change in a particular azimuthal mode, but rather to a changes across all of the acoustically important azimuthal modes. This is explored in the following section via a stability analysis of the main azimuthal modes. 

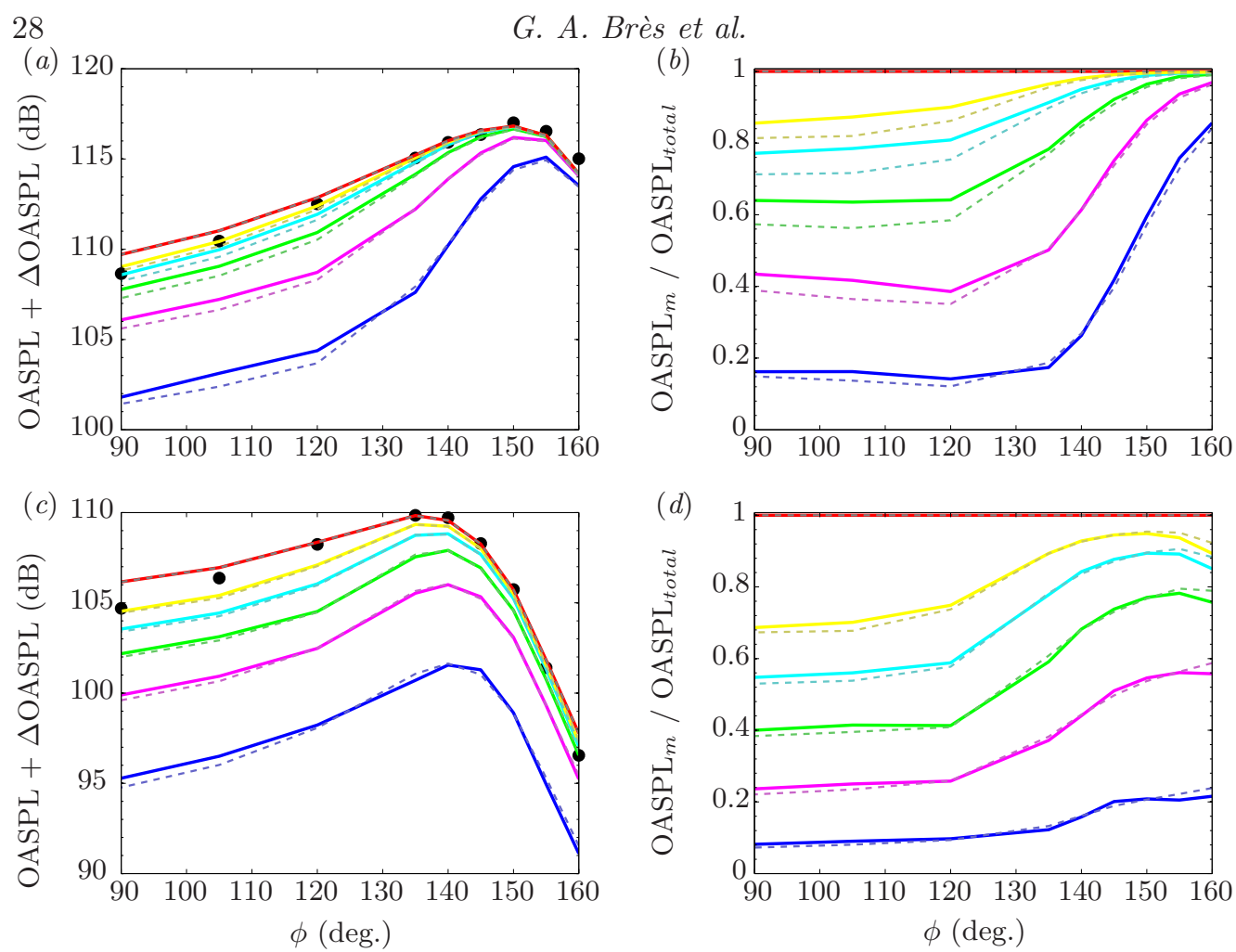

Figure 20. (Colour online) Azimuthal mode analysis of the shifted OASPL directivity and the modes relative OASPL contribution, computed over the frequency range $(a, b) 0.05 \leqslant S t \leqslant 3$ and $(c, d) 1 \leqslant S t \leqslant 3$ for the experimental data (symbols), initially laminar jet 10M (dashed lines) and turbulent jet BL16M_WM_Turb (solid lines): ( • , - $)$ total (i.e., all modes); ( - ) mode $m=0 ;($ ) modes $m=0$ to $1 ;($ ( $) m=0$ to 2 ; ( - ) $m=0$ to 3 ; ( -) $m=0$ to 4 .

\subsection{Linear stability analysis}

Large-scale turbulent structures in jets are often modelled using linear stability analysis, using the jet mean profiles as base flows upon which linearization of the governing equations is performed. The inflectional velocity profile of the shear layer leads to the Kelvin-Helmholtz instability, and disturbances have exponential spatial growth near the nozzle. Further downstream the Kelvin-Helmholtz mode becomes stable; the resulting overall solution is a wavepacket (Crighton \& Gaster 1976). Earlier modelling work was based on comparisons with artificially excited jets (Cohen \& Wygnanski 1987; Petersen \& Samet 1988), but with modern experimental methods, it is now feasible to compare wavepackets from linear stability theory with experimental data of turbulent jets without artificial periodic forcing. Suzuki \& Colonius (2006) and Gudmundsson \& Colonius (2011) presented linear stability results in good agreement with the near pressure field of subsonic jets, and Cavalieri et al. (2013) have extended the comparison to the velocity field, with similar agreement. A review of methods and main results can be found in Jordan \& Colonius (2013).

In what follows, linear stability theory is used to evaluate the differences between jets with initially laminar and initially turbulent shear layers, and in particular, to further probe the reasons for the over-prediction of high-frequency noise from laminar jets. Focus is given on the spatial growth rate of the Kelvin-Helmholtz mode. Disturbances are assumed to have an implicit $\exp [\mathrm{i}(\alpha x-\omega t)]$ dependence, with $\alpha=\alpha_{r}+\mathrm{i} \alpha_{i}$ a complex- 
valued wavenumber. In the local spatial stability problem, the frequency $\omega$ is given, and the wavenumber $\alpha$ becomes an eigenvalue of the problem. Spatial growth rates, related to exponential amplification in $x$, are given by $-\alpha_{i}$. The different mean flows of initially laminar and initially turbulent jets are expected to lead to differences in growth rates, which are explored next.

\subsubsection{Methodology}

Linear stability analysis is conducted for the mean flows extracted from both the simulation 10M and BL16M_WM_Turb, whose initial shear layers are laminar and turbulent, respectively. Near-nozzle mean velocity profiles were taken as locally-parallel base flows and the analysis focuses on the main azimuthal modes, $m=0$ to 4 . Solutions of the compressible Rayleigh equation (Michalke 1984) were obtained using a pseudo-spectral method (Trefethen 2000). To ensure that a sufficiently high number of Chebyshev nodes was used in the discretisation of the velocity profile, the mapping function of (Lesshafft \& Huerre 2007) was used, with the same parameters used in the article.

The use of differentiation matrices based on Chebyshev polynomials require an accurate representation of the base flow on the numerical nodes, which is of particularly concern near the nozzle, where mean-flow gradients are high. A usual solution is to use a functional fit to the mean profiles. To maintain consistency with stability analyses focusing on the near-nozzle region (Fontaine et al. 2015; Sasaki et al. 2017), we have chosen to fit the mean velocity profiles from the LES, using an expression similar to the one used by Fontaine et al. (2015), given as

$$
U(r)=\frac{M}{4}\left[1-\tanh \left(\frac{r_{o}}{4 \theta_{1}}\left[\frac{r}{r_{o}}-\frac{r_{o}}{r}\right]\right)\right]\left[1-\tanh \left(\frac{r_{o}}{4 \theta_{2}}\left[\frac{r-r_{s}}{r_{o}}-\frac{r_{o}}{r}\right]\right)\right]
$$

with parameters $r_{o}, r_{s}, \theta_{1}$ and $\theta_{2}$ determined from a least-squares fit.

The thin shear layers in the near-nozzle velocity profile make it difficult to obtain numerical convergence of the stability results, and require a high number of Chebyshev polynomials in the discretisation. It was verified that results obtained using 550 and 600 Chebyshev polynomials led to nearly identical results for the range of frequencies and azimuthal wavenumbers studied here. Thus, the $N=600$ discretisation is used for the analysis of the near-nozzle region (up to $x / D=0.25$ ). Further downstream, a discretisation with $N=400$ was sufficient for convergence.

\subsubsection{Comparison of the stability of initially laminar and turbulent jets}

Figure 21 shows the growth rates of the Kelvin-Helmholtz mode for various near-nozzle axial stations of the initially laminar jet (case 10M) and the initially turbulent jet (case BL16M_WM_Turb). The jet with initially turbulent shear layer has a broader $S t$ range of Kelvin-Helmholtz instability for the station closest to the nozzle, at $x / D=0.08$. However, the downstream evolution of the shear-layer thickness is faster for the turbulent jet, and when the analysis is carried out at downstream positions, the range of unstable $S t$ and the maximum growth rates are quickly reduced for the turbulent jet (see figure 21(b)). The initially laminar jet has a slower shear-layer development, and thus the growth rates have only modest changes with $x / D$. As a result, from $x / D=0.2$ the situation is reversed, and the initially laminar jet has a broader range of unstable Strouhal numbers, and higher maximum growth rates.

For the stations close to the nozzle exit, the results for the first 5 dominant modes identified by the azimuthal decomposition analysis in $\S 4.2$ are nearly identical, as shown in figure 21. For the thin shear layers in the near-nozzle region, the azimuthal wavenum- 
30

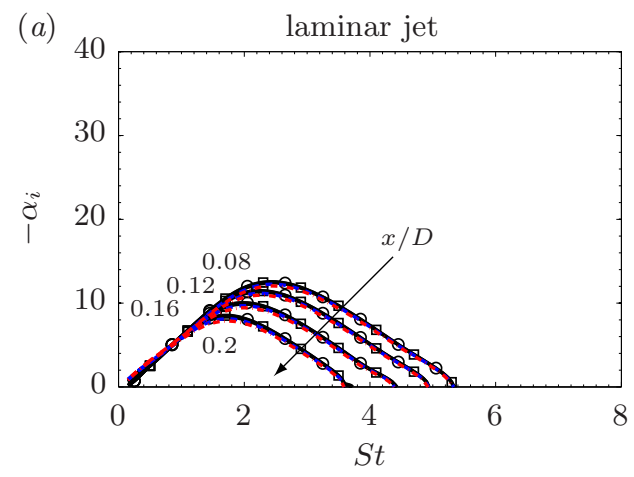

G. A. Brès et al.

(b)

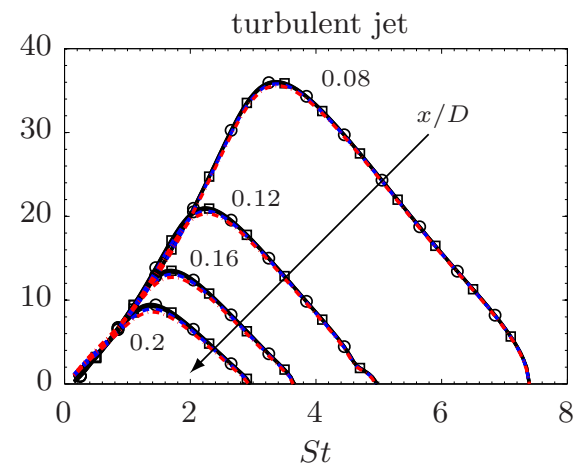

Figure 21. (Colour online) Growth rates of the Kelvin-Helmholtz instability for azimuthal modes $m=0(\boldsymbol{\longrightarrow}), m=1(-\circ-), m=2(-\square-), m=3(---)$ and $m=4(-\cdot-)$ for near-nozzle axial stations at $x / D=0.08$ to 0.2 : (a) initially laminar jet (case $10 \mathrm{M}$ ); $(b)$ initially turbulent jet (case BL16M_WM_Turb).

ber for these modes is still much larger than the shear-layer thickness, and there is no significant $m$ dependence for azimuthal modes between 0 and 4 .

Figure 22 shows a comparison of the growth rates of the Kelvin-Helmholtz mode at axial stations further downstream of the nozzle. Here, the shear-layer is thicker and the differences between modes become more apparent but the same trend is observed for all the modes considered. At $x / D=0.25$, the growth rates of the two jets are close for Strouhal numbers up to 1 . Above that value, the initially laminar jet has higher amplification rates due to its thinner shear layer at that station. Thus, the persistence of a thin, transitional shear layer in the near-nozzle region in the 10M case leads to a persistence of the spatial amplification of the Kelvin-Helmholtz mode for $S t>1$ compared to the turbulent jet for case BL16M_WM_Turb. Such sustained growth rates in the 10M simulation are likely the cause of the over-estimation of sound radiation for $S t>1$ in figures 9 and 15 , and of the higher RMS levels in the near-nozzle region in figure 11, compared to the turbulent jet simulations and to experiments.

Once the shear layer transitions to turbulence in the $10 \mathrm{M}$ simulation, the faster increase of momentum thickness shown in figure 12 progressively reverses these trends, limiting the growth of the Kelvin-Helmholtz mode. At $x / D=0.5$, growth rates are similar for the two simulations, and at $x / D=0.75$, the initially turbulent jet starts to present slightly larger growth rates than the initially laminar one; this can be related to the reversal in momentum thickness shown in figure 12 , with the initially turbulent jet now with a thinner shear layer.

\section{Conclusions}

Large-eddy simulations and experimental measurements of an isothermal Mach 0.9 turbulent jet were performed in order to investigate the role of the nozzle-interior boundary layers, and to generate databases for jet noise analysis and modelling. For all cases considered, the nozzle geometry was explicitly included in the computational domain using unstructured body-fitted grids, with mesh size ranging from 10 to 69 million control volumes. All the simulations were carried out at the experimental operating condition, including the full diameter-based Reynolds number $R e \approx 1 \times 10^{6}$.

In the LES, an effort was made to obtain initially turbulent jet matching the experimental conditions. First, a preliminary parametric study was performed to quantify the 

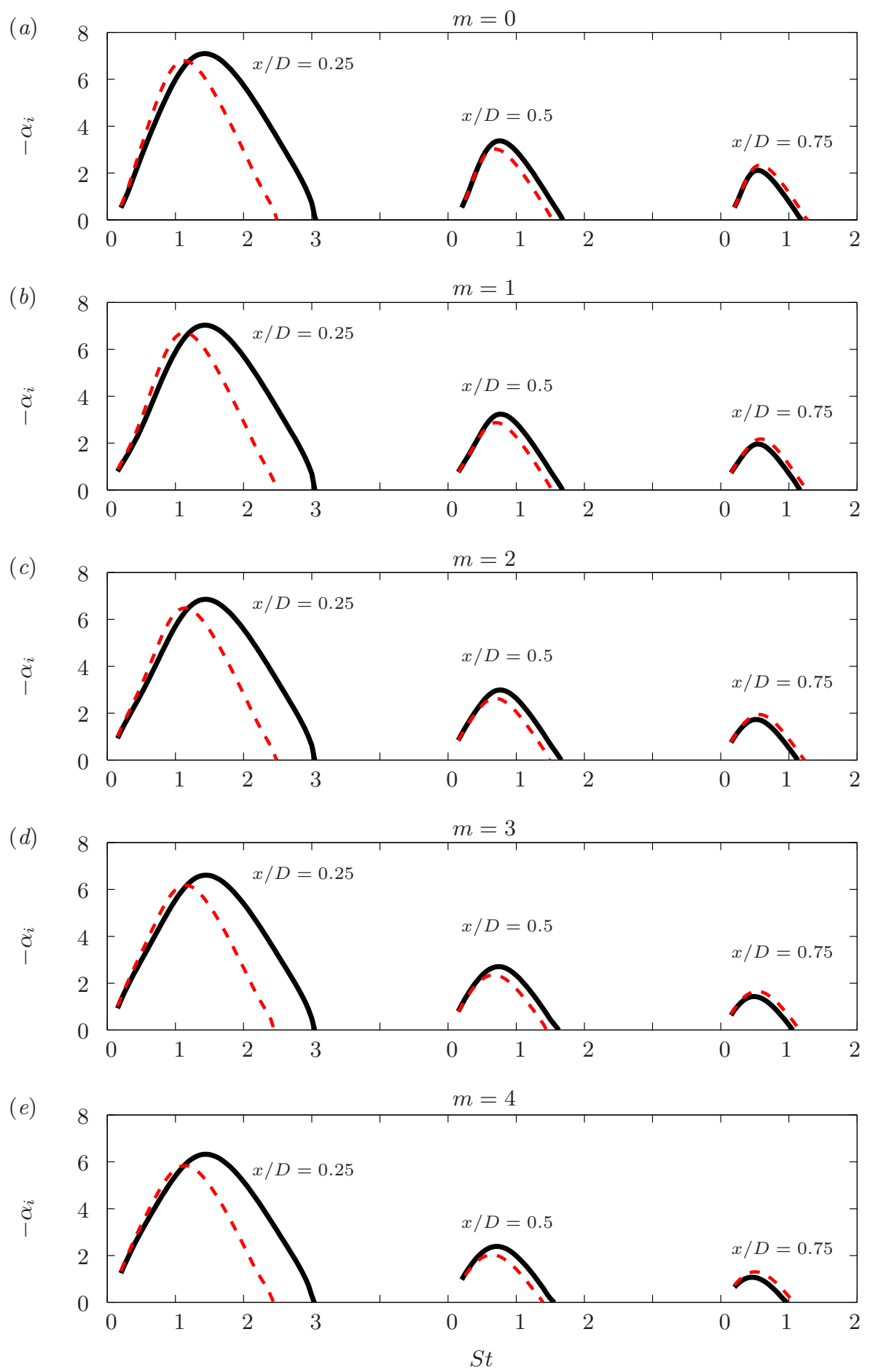

Figure 22. (Colour online) Growth rates of the Kelvin-Helmholtz instability for azimuthal modes $m=0$ to $4(a-e)$ for axial stations at $x / D=0.25$ to $0.75:(-)$ initially laminar jet (case 10M) and (--- ) initially turbulent jet (case BL16M_WM_Turb). 
separate and combined effects of the different aspects of the nozzle-interior turbulence modelling problem. These included localized adaptive mesh refinement inside the nozzle, application of synthetic turbulence to model the boundary trip present in the experiment, and wall modelling. In terms of ranking in importance, the near-wall grid refinement had the most significant and beneficial impact on the flow and far-field sound predictions, followed by wall modelling and lastly synthetic turbulence. While the addition of low levels of synthetic turbulence had limited effects, higher initial levels lead to an increase in pressure fluctuations and core turbulence inside the nozzle, and to an excess of highfrequency radiated noise. Here, the parametric study on the synthetic turbulence inputs is limited to two different fluctuation amplitudes, with the choices of the fluctuation length scale and time scale rooted in physics. Future work could explore in more details the influence of these parameters on the flow and noise results, or the use of geometrical tripping approach as an alternative to the synthetic turbulence method. In terms of performance, the refinement inside the nozzle lead to only a small increase in grid size (i.e, about 5 million control volumes), independent of the resolution in the jet plume and no change in the simulation time step. This resulted in a modest increase of computational cost when all the different modellings were used.

Overall, the results showed the best match with experiments when all three methods were applied inside the nozzle, compared to the typical approach based on coarse resolution in the nozzle and laminar flow assumption. With nozzle-interior turbulence modelling, the nozzle-exit velocity statistics exhibited fully turbulent profiles similar to the experimental data, and the far-field noise spectra closely matched the measurements, within $0.5 \mathrm{~dB}$ for the relevant angles and frequencies. In contrast, the initially laminar jet featured systematic over-prediction of the high-frequency far-field noise, consistent with previous experimental studies of (nominally) laminar versus turbulent jets (Brown \& Bridges 2006; Zaman 2012; Karon \& Ahuja 2013).

Independently of the nozzle-interior turbulence modelling, all of the simulations contained discrete tones in the pressure spectra inside the nozzle and in the near-nozzle region. These spectral peaks are related to acoustic waves that are trapped within the jet potential core and decay rapidly away from the jet (Towne et al. 2017; Schmidt et al. 2017). The tones were therefore observed in the simulations and the companion experiments in the near-field acoustic pressure close to the nozzle exit, and not in the far-field noise.

For both initially laminar and turbulent jets, decomposition of the radiated noise in azimuthal Fourier mode was performed. The analysis showed that the two jets have similar azimuthal characteristics and that the axisymmetric mode $m=0$ was dominant for the main frequency range $0.05 \leqslant S t \leqslant 0.5$ at peak radiation angles $\phi=140^{\circ}$ to $160^{\circ}$. At these angles, the first 3 Fourier azimuthal modes of the LES data recovered more that $97 \%$ of the total acoustic energy and more than $65 \%$ (i.e., error less than $2 \mathrm{~dB}$ error) over all angles.

Linear stability analysis of the near-nozzle mean velocity profiles was then conducted in both jets, focusing on the dominant modes identified by the azimuthal decomposition analysis. The results suggested that the differences in radiated noise observed between the initially laminar and turbulent jets for $S t>1$ are related to the differences in growth rate of the Kelvin-Helmholtz mode in the near-nozzle region at these frequencies. This conclusion holds for all the azimuthal modes considered, up to $m=4$. Here, the lack of significant dependence on azimuthal modes is likely due to the fact that the azimuthal wavenumber of the dominant modes is much larger than the shear-layer thickness close to the nozzle for both jets. 


\section{Acknowledgments}

The LES studies were supported by NAVAIR SBIR project under the supervision of J.T. Spyropoulos, with computational ressources provided by DoD HPCMP at the ERDC DSRC supercomputer facility. G.A.B. would like to thank the main architects of the Charles solver, F. Ham and S.T. Bose, for their contributions to the LES studies. P.J. acknowledges the support of the French National Research Agency (ANR) through the project COOLJAZZ. Part of this work was performed during the Center for Turbulence Research Summer Program 2014 at Stanford University. The authors also thank S. Piantanida for performing the experimental measurements reported in figures 17 and 24 .

\section{Appendix A. Experimental set-up}

The study considers an isothermal subsonic jet issued from a contoured convergent-straight round nozzle of exit diameter $D=0.05 \mathrm{~m}$. The experiments were performed at the Bruit \& Vent jet-noise facility of the Institut PPRIME, Poitiers, France, whose anechoïc chamber measures $9.6 m \times 6 m \times 3.4 m$ and is equipped with absorbing foam wedges that provide anechoïc conditions down to $212 \mathrm{~Hz}$. The flow is driven by a rotary screw compressor, downstream of which it passes through a series of regulation valves and a heat exchanger that permit manipulation of flow rate and temperature. From the heat exchanger the flow travels through $17 \mathrm{~m}$ of acoustically lined piping, at velocities of order $\mathcal{O}(5 \mathrm{~m} / \mathrm{s})$, before being delivered to a settling chamber that contains flow conditioning comprised of a honey-comb structure followed by two wire meshes. Downstream of these, the settling chamber is equipped with temperature and pressure sensors that provide signals to a PID controller that ensures constant operating conditions. For the study considered here, these were defined in terms of the nozzle-pressure ratio, $N P R=P_{t} / P_{\infty}=1.7$ and nozzle temperature ratio, $N T R=T_{t} / T_{\infty}=1.15$, where the subscripts $t$ and $\infty$ refer, respectively, to stagnation (total) and free-stream values. The PID controller maintained the flow at constant Mach number, $M_{j}=U_{j} / c_{j}=0.9$ (to within $\pm 0.01 M$ ) and constant temperature ratio, $T_{j} / T_{\infty}=1$ (to within, $\left.\pm 0.01 T_{j} / T_{\infty}\right)$, where $T_{j}$ and $U_{j}$ are the mean jet temperature and streamwise velocity at the exit and $c$ is the speed of sound. With these conditions, the Reynolds number is $R e=\rho_{j} U_{j} D / \mu_{j} \approx 1 \times 10^{6}$. The settling chamber transitions to a cylindrical nozzle via a $7: 1$ area contraction.

Boundary layer transition is forced by means of an azimuthally homogeneous carborundum strip. The carborundum particles, of diameter $0.005 D$, are glued to the internal nozzle wall. The glued carborundum composite presents a thickness of $0.0064 D$ to the oncoming flow and extends $0.28 D$ in the streamwise direction. The downstream edge of the strip is situated approximately $2.5 \mathrm{D}$ upstream of the nozzle exit.

Three microphone arrays were used to perform pressure measurements. A 48-microphone near-field cage array provides access to, and azimuthal Fourier decomposition of, the hydrodynamic nearfield on a conical surface surrounding the jet; an axially traversable, 18-microphone, azimuthal array provides measurement, and azimuthal Fourier decomposition, of the sound field on a cylindrical surface of radius, $r=14.3 D$ and axial span, $0 \leqslant x / D \leqslant 40$; a polar array centered on the jet exit, of radius $R=50 D$, comprised of a single microphone every $10^{\circ}$ in the range $90^{\circ} \leqslant \phi \leqslant 160^{\circ}$, where the jet inlet angle 


$\begin{array}{ccc}\text { (a) } \mathbf{x} / \mathbf{D} & \mathbf{r} / \mathbf{D} & \phi \\ 0.12 & 0.72 & 99.5^{\circ} \\ 2.00 & 0.98 & 153.9^{\circ} \\ 2.62 & 1.07 & 157.8^{\circ} \\ 3.42 & 1.18 & 160.1^{\circ} \\ 4.47 & 1.33 & 163.4^{\circ} \\ 5.85 & 1.52 & 165.4^{\circ} \\ 7.65 & 1.78 & 166.9^{\circ} \\ 10 & 2.10 & 168.1^{\circ}\end{array}$

\begin{tabular}{|c|c|c|}
\hline $\mathrm{x} / \mathrm{D}$ & $r / D$ & $\phi$ \\
\hline & 14.3 & 90 \\
\hline 3.83 & 14.3 & 105 \\
\hline 8.25 & 14.3 & 120 \\
\hline 14.30 & 14.3 & 135 \\
\hline 17.04 & 14.3 & 140 \\
\hline 20.42 & 14.3 & 145 \\
\hline 24.77 & 14.3 & 150 \\
\hline 30.66 & 14.3 & 155 \\
\hline 39.29 & 14.3 & \\
\hline
\end{tabular}

(c) $\mathbf{x} / \mathbf{D} \quad \mathbf{r} / \mathbf{D} \quad \phi$

$\begin{array}{lll}0 & 50.00 & 90^{\circ}\end{array}$

$8.68 \quad 49.24 \quad 100^{\circ}$

$\begin{array}{llll}17.10 & 46.98 & 110^{\circ}\end{array}$

$\begin{array}{llll}25.00 & 43.30 & 120^{\circ}\end{array}$

$\begin{array}{llll}32.14 & 38.30 & 130^{\circ}\end{array}$

$38.3032 .14 \quad 140^{\circ}$

$\begin{array}{llll}43.30 & 25.00 & 150^{\circ}\end{array}$

$46.98 \quad 17.10 \quad 160^{\circ}$

TABLE 4. Coordinates $x-r$ and corresponding jet inlet angle $\phi$ of the microphones for $(a)$ the near-field cage array, $(b)$ the cylindrical array, and, $(c)$ the polar array.

$\phi$ is measured from the upstream jet axis, is used to provide directivity information. All the microphone coordinates are provided in table 4. Details about the noise post-processing procedure and non-dimensionalization are presented in Appendix B.

The velocity field was probed using hot-wire anemometry and particleimage velocimetry (PIV). The hot-wire had diameter $2.5 \mu \mathrm{m}$ and length $0.7 \mathrm{~mm}$ and was used in conjunction with a Dantec 55M01 anemometer; the corner frequency of the set-up was $60 \mathrm{kHz}$, corresponding to Strouhal number, $S t \approx$ 10. Measurements were performed immediately downstream of the nozzle lip, at $x / D=0.04$ and over the radial range $0.35 \leqslant r / D \leqslant 0.55$ in order to obtain the signature of the exit boundary layer.

The PIV system consisted of a Photron SAZ camera and a $532 \mathrm{~nm}$ Continuum MESA PIV laser providing $6 \mathrm{~mJ}$ of light-pulse energy. The system was placed on a traverse parallel to the jet-axis so as to scan the flow field over the streamwise extent, $0 \leqslant x / D \leqslant 20$. The cameras were equipped with $100 \mathrm{~mm}$ Macro lenses with low optical distortion and aperatures set at $f \# 4$. Two fields of view (FOV) were used, the first had measurement area, $2 D \times 2 D$, and was used over the axial range $0 \leqslant x / D \leqslant 6$. The measurement area of the second was $4 D \times 4 D$ and it was used over the range $5<x / D \leqslant 20$. With this configuration a finer spatial resolution is obtained in the region up to the end of the potential core, where velocity gradients are greatest. The entire measurement ensemble comprised 11 FOVs over the said axial range, with a $20 \%$ overlap between these in order to ensure correct alignment of the measured fields. Calibration was performed at each acquisition station in order to correct for optical distortions and laser-sheet/measurement-plane misalignments using a self-calibration procedure(Wieneke 2005).

Both the jet flow and the surrounding air were seeded with glycerin vapour particles, whose diameters lie in the range $1-2 \mu \mathrm{m}$, ensuring that they follow the velocity fluctuations of interest in the study. A velocity histogram analysis showed that no peak-locking occured. The image acquisitions were performed at $20 \mathrm{kHz}(10000 \mathrm{PIV}$ samples per second) at a resolution of $1024 \times 1024$ pixels. The time between the two laser pulses, set according to the local velocity amplitude and the laser-sheet width (set at $2 \mathrm{~mm}$ ), ranged between 4 and $5 \mu s$. Each acquisition comprised 42000 image pairs. PIV calculations were performed using LaVision software, Davis 8.2 and a multi-pass iterative PIV algorithm with deforming interrogation areas was used to account for the 
local mean velocity gradients (Scarano 2002). The PIV interrogation area was set to $32 \times 32$ pixels for the first pass, and decreased to $16 \times 16$ pixels for the remaining passes, with a $50 \%$ overlap between neighbouring interrogation areas. Computed displacements were only retained for correlation-peak ratios above 1.3. After each pass a Universal Outlier Detection (UOD) was applied on a $3 \times 3$ grid to eliminate corrupted data and to enhance the particle-motion calculation (Westerweel \& Scarano 2005). Finally, prior to computation of flow statistics, a 5-sigma filter was applied to remove the remaining outliers which were replaced using the UOD technique.

\section{Appendix B. Noise post-processing procedure}

The experimental data is acquired at a frequency of $f_{a c}=200 \mathrm{kHz}$ (which corresponds to a Strouhal number, $S t_{a c}=f_{a c} D / U_{j}=32$ ). The acquisition runs for 20 seconds, and so each signal has $N=4 \times 10^{6}$ points.

The mean value is first removed:

$$
p(t)=P(t)-\frac{1}{N} \sum_{t=1}^{N} P(t) .
$$

Prior to down sampling the data is low-pass filtered via convolution with a three-point Gaussian, $w=[0.25 ; 0.5 ; 0.25]$ :

$$
p_{L P}(t)=w(1) p(t-1)+w(2) p(t)+w(3) p(t+1) .
$$

This time-domain low-pass filter ensures that the down-sampled data is not aliased; the data is down-sampled at $f_{u}=100 \mathrm{kHz}\left(S t_{u}=16\right)$ and spectra are computed using this data.

Fast Fourier Transforms (FFT) are performed on blocks of data of size $N_{f f t}=2048$, and an overlap of $75 \%$ is imposed; i.e. block $i$, is

$$
N b_{i}=p_{L P}\left(1+(i-1) N_{o v}\right): p_{L P}\left(N_{f f t}+(i-1) N_{o v}\right),
$$

where

$$
N_{o v}=\frac{N_{f f t}}{4}-1
$$

A Hanning window is applied to each block prior to application of the FFT. A Fourier-transformed block is thus:

$$
\hat{p}(f)=\frac{\sqrt{8 / 3}}{N_{f f t}}\left(F F T\left(H(t) . * p_{L P}(t)\right)\right),
$$

where the factor $\sqrt{8 / 3}$ corrects for the energy loss associated with the Hanning window. The power spectral density of block $i$ is then computed as:

$$
\hat{P}_{i}(f)=\frac{2}{\Delta f} \hat{p}_{i}\left(f_{1}: \Delta f: f_{N y q}\right) \hat{p}_{i}^{*}\left(f_{1}: \Delta f: f_{N y q}\right),
$$

where $\Delta f=49 \mathrm{~Hz}(\Delta S t \approx 0.008), f_{N y q}=50 \mathrm{kHz}(S t \approx 8)$ and $\hat{p}_{i}^{*}(f)$ is the complex conjugate of $\hat{p}_{i}(f)$. The averaged narrowband power spectral density (PSD) is then

$$
\hat{P}(f)=\frac{1}{N b} \sum_{i=1}^{N b} \hat{P}_{i}(f),
$$


where $N b=3903$ is the total number of blocks of data.

In order for comparisons between simulations and experiments, or between experiments and experiments for that matter, to be valid, it is necessary to use appropriate non-dimensionalisation. The absolute jet conditions in the wind tunnel can vary from day to day, as the target control parameters are the dynamic Mach number, $M_{j}=U_{j} / c_{j}=0.9$ (as opposed to the acoustic Mach number, $M_{a}=U_{j} / c_{\infty}$ ) and a unitary temperature ratio, $T_{j} / T_{\infty}=1$. The ambient temperature in the anechoïc chamber, not being controlled, varies, as do, consequently, the jet velocity and temperature.

The following non-dimensionalisation of the measured data is thus performed. The power spectral density $\hat{P}(f)$ is first scaled so as to account for changes in the dynamic head of the jet:

$$
\frac{\hat{P}(f)}{\rho_{j}^{2} U_{j}^{4}}
$$

This quantity has units of $s^{-1}$, and its frequency dependence can be made non-dimensional by means of the factor $\frac{U_{j}}{D}$, (the power spectral density is now a function of the Strouhal number, $\left.S t=\frac{f D}{U_{j}}\right)$ :

$$
\frac{\hat{P}(f)}{\rho_{j}^{2} U_{j}^{4}} \times \frac{U_{j}}{D}
$$

As this quantity is non-dimensional, calculation of the PSD in $d B / S t$ must be performed by means of a non-dimensional reference acoustic pressure level:

$$
P_{r e f}=\frac{P_{r e f}^{*}}{\rho_{\infty} c_{\infty}^{2}}
$$

where $P_{r e f}^{*}=20 \mu \mathrm{Pa}$ is the dimensional reference pressure. We thus have

$$
P S D=10 \log _{10}\left[\frac{\frac{\hat{P}(f)}{\rho_{j}^{2} U_{j}^{4}} \times \frac{U}{D}}{P_{r e f}^{2}}\right]=10 \log _{10}\left[\frac{\hat{P}(f)}{P^{* 2}{ }_{r e f}^{2}} \times \frac{U_{j}}{D} \times \frac{\rho_{\infty}^{2} c_{\infty}^{4}}{\rho_{j}^{2} U_{j}^{4}}\right]
$$

In the case of an isothermal jet, $\rho_{j} / \rho_{\infty}=1$, and $M_{a}=M_{j}$, such that the expression reduces to:

$$
P S D=10 \log _{10}\left[\frac{\hat{P}(f)}{P_{r e f}^{* 2}} \frac{U_{j}}{D} \frac{1}{M_{a}^{4}}\right]
$$

\section{Appendix C. Trapped acoustic waves in the jet potential core}

Towne et al. (2017) and Schmidt et al. (2017) recently identified and explained a new class of acoustic waves that are trapped within the potential core of subsonic jets. These waves experience the shear-layer as a pressure-release duct and are therefore radially confined to the near-field of the jet. At certain frequencies, the trapped waves resonate due to repeated reflection between end conditions provided by the nozzle-exit plane and the streamwise contraction of the potential core. This resonance leads to an accumulation of energy that can be observed as discrete tones in near-field spectra.

Simple linear models of the trapped waves suggest that conditions for resonance, namely the existence of both upstream and downstream propagating trapped waves, exist 


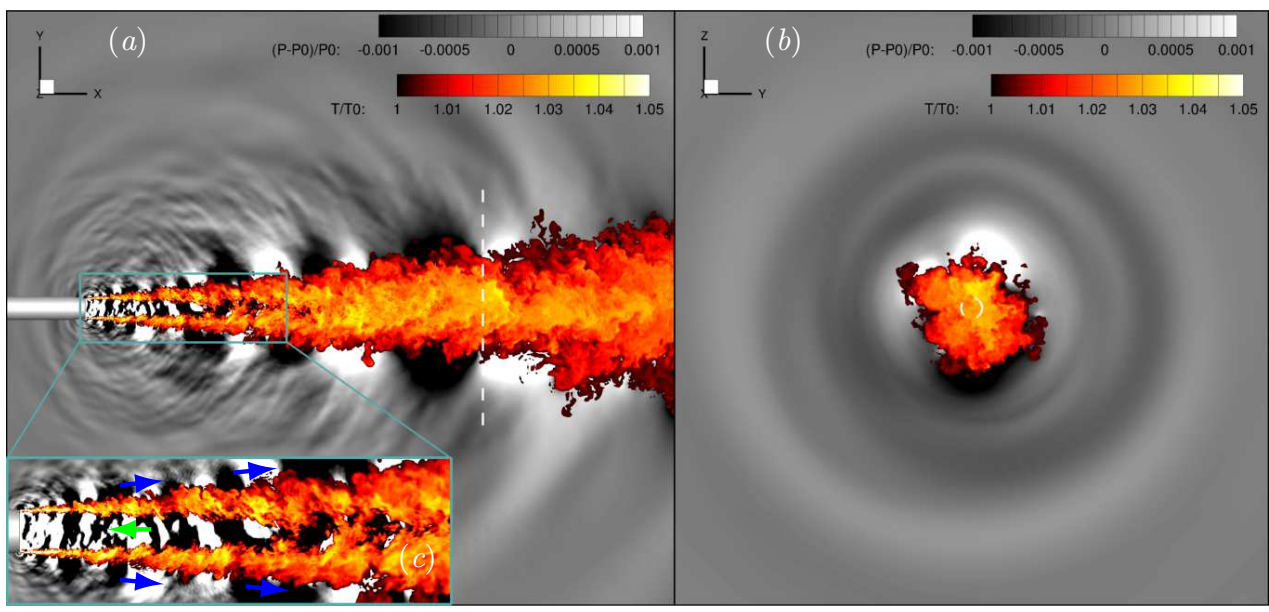

Figure 23. (Colour online) Instantaneous pressure and temperature field for the case BL69M_WM_Turb $(a)$ in the mid-section of the jet plume, $(b)$ at the cross-section $x / D=20$ (as indicated by the vertical white dashed line in $(a))$, and $(c)$ in the potential core. The nozzle external surface is shown in metallic grey and the white dashed circle represents the outline of the nozzle lip. The green and blue arrows indicate the upstream-propagating trapped waves and downstream-propagating acoustic waves, respectively.
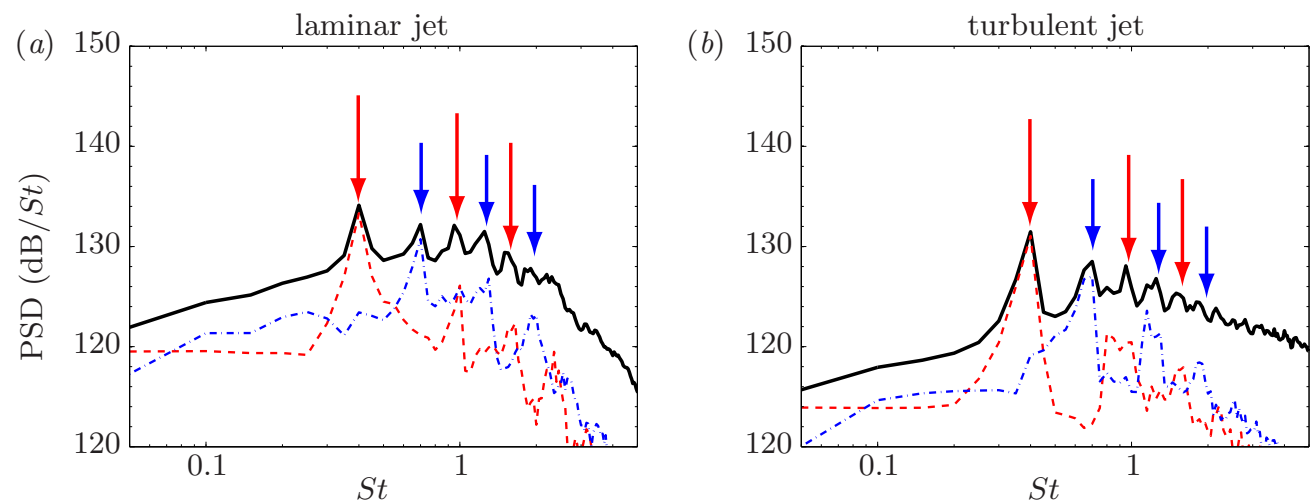

Figure 24. (Colour online): Power spectra density and azimuthal mode decomposition of the pressure fluctuations in the near-nozzle region at $(x / D, r / D)=(0.12,0.72)$ for $(a)$ the initially laminar jet 10M and $(b)$ the initially turbulent jet BL16M_WM_Turb: ( - ) total (i.e., all modes); ( --- ) $m=0 ;(--$ ) $m=1$. The arrows indicate the frequencies of the trapped acoustic waves predicted in Towne et al. (2017)

only for jets with certain Mach numbers and temperature ratios (Towne et al. 2017). For isothermal jets, the resonating waves and associated tones are expected to be observed for Mach numbers in the range $0.82<M_{j}<1$. The jet condition considered in this paper falls within this range, and the resulting tones were pointed out in figures 8 and 17 . The trapped acoustics waves are also visible in animations of the instantaneous pressure field in the jet potential core, in particular the upstream propagating waves, as indicated by the green arrow in figure 23 (see supplementary movie). The flow visualization at the cross-section $x / D=20$ in figure $23(b)$ also further highlights the axisymmetric nature of the far-field noise radiation previously discussed in the azimuthal mode decomposition analysis in $\S 4.2$.

One property of the trapped waves is that each resonant frequency is associated with 
one specific azimuthal wavenumber. This provides a straightforward way of confirming that the tones have been properly identified. Figure 24 shows the pressure spectra as well as its first two azimuthal components at the same location $(x / D, r / D)=(0.12,0.72)$ previously reported in figure $17(a)$. For both laminar and turbulent jets, the peaks observed in the total spectra clearly correspond to a particular azimuthal mode. Furthermore, the frequency-azimuthal wavenumber combination of each peak matches the predictions provided by the analytical vortex-sheet model in Towne et al. (2017), confirming that these peaks can indeed be attributed to resonating acoustic waves in the jet core.

\section{REFERENCES}

Andersson, N., Eriksson, L. E. \& Davidson, L. 2005 Effects of inflow conditions and subgrid model on LES for turbulent jets. AIAA Paper 2005-2925.

BODART, J. \& LARsson, J. 2011 Wall-modeled large eddy simulation in complex geometries with application to high-lift devices. In Annual Research Briefs. Center for Turbulence Research, Stanford University.

Bodony, D. J. \& Lele, S. K. 2005 On using large-eddy simulation for the prediction of noise from cold and heated turbulent jets. Phys. Fluids 17(085103).

Bodony, D. J. \& Lele, S. K. 2008 Current status of jet noise predictions using large-eddy simulation. AIAA J. 46, 346-380.

Bogey, C. \& BAilly, C. 2005 Effects of inflow conditions and forcing on subsonic jet flows and noise. AIAA J. 43 (5), 1000-1007.

Bogey, C. \& BAilly, C. 2010 Influence of nozzle-exit boundary-layer conditions on the flow and acoustic fields of initially laminar jets. J. Fluid Mech. 663, 507-538.

Bogey, C., Barré, S. \& BAilly, C. 2008 Direct computation of the noise generated by subsonic jets originating from a straight pipe nozzle. International Journal of Aeroacoustics 7 (1), 1-21.

Bogey, C. \& Marsden, O. 2016 Simulations of initially highly disturbed jets with experimentlike exit boundary layers. AIAA J. 54, 1299-1312.

Bogey, C., Marsden, O. \& BAilly, C. 2011 Large-eddy simulation of the flow and acoustic fields of a reynolds number $10^{5}$ subsonic jet with tripped exit boundary layers. Phys. Fluids 23(035104).

Bogey, C., Marsden, O. \& Bailly, C. 2012 Influence of initial turbulence level on the flow and sound fields of a subsonic jet at a diameter-based reynolds number of $10^{5}$. J. Fluid Mech. 701, 352-385.

Bradshaw, P., Ferriss, D. H. \& Johnson, R. F. 1964 Turbulence in the noise-producing region of a circular jet. J. Fluid Mech. pp. 591-624.

Brès, G. A., Bose, S. T., Ham, F. E. \& Lele, S. K. 2014 Unstructured large eddy simulations for nozzle interior flow modeling and jet noise predictions. AIAA paper 2014-2601.

Brès, G. A., Ham, F. E., Nichols, J. W. \& Lele, S. K. 2013 Nozzle wall modeling in unstructured large eddy simulations for hot supersonic jet predictions. AIAA paper 20132142.

Brès, G. A., Ham, F. E., Nichols, J. W. \& Lele, S. K. 2017 Unstructured large eddy simulations of supersonic jets. AIAA J. 55 (4), 1164-1184.

Brès, G. A., Jaunet, V., Le Rallic, M., Jordan, P., Colonius, T. \& Lele, S. K. 2015 Large eddy simulation for jet noise: the importance of getting the boundary layer right. AIAA paper 2015-2535.

Brès, G. A., Jaunet, V., Le Rallic, M., Jordan, P., Towne, A., Schmidt, O. T., Colonius, T., Cavalieri, A. V. G. \& Lele, S. K. 2016 Large eddy simulation for jet noise: azimuthal decomposition and intermittency of the radiated sound. AIAA paper 2016-3050.

Bridges, J. E. \& Hussain, A. K. M. F. 1987 Roles of initial conditions and vortex pairing in jet noise. J. Sound Vib. 117(2), 289-331. 
Brown, C. \& Bridges, J. 2006 Small hot jet acoustic rig validation. Tech. Rep. TM 2006214234. NASA.

Bühler, S., Kleiser, L. \& Bogey, C. 2014a Simulation of subsonic turbulent nozzle jet flow and its near-field sound. AIAA J. 52 (8), 1653-1669.

BüHler, S., Obrist, D. \& Kleiser, L. $2014 b$ Laminar and turbulent nozzle-jet flows and their acoustic near-field. Phys. Fluids 26, 086103.

Cavalieri, A. V. G., Daviller, G., Comte, P., Jordan, P., Tadmor, G. \& Gervais, Y. 2011 Using large eddy simulation to explore sound-source mechanism in jets. J. Sound Vib. 330, 4098-4113.

Cavalieri, A. V. G., Jordan, P., Colonius, T. \& Gervais, Y. 2012 Axisymmetric superdirectivity in subsonic jets. J. Fluid Mech. 704, 388-420.

Cavalieri, A. V. G., Rodríguez, D., Jordan, P., Colonius, T. \& Gervais, Y. 2013 Wavepackets in the velocity field of turbulent jets. J. Fluid Mech. 730, 559-592.

Chor, H. \& Moin, P. 2012 Grid-point requirements for large eddy simulation: Chapman's estimates revisited. Phys. Fluids 24(011702).

Cohen, J. \& Wygnanski, I. 1987 The evolution of instabilities in the axisymmetric jet. part 1. the linear growth of disturbances near the nozzle. Journal of Fluid Mechanics 176, 191-219.

Crighton, D. G. \& Gaster, M. 1976 Stability of slowly diverging jet flow. J. Fluid Mech. 88 (2), 397-413.

Ffowcs Williams, J. E. \& Hawkings, D. L. 1969 Sound generation by turbulence and surfaces in arbitrary motion. Philosophical Transactions of the Royal Society of London. Series A, Mathematical and Physical Sciences 264, 321-342.

Fontaine, R. A., Elliot, G. S., Austin, J. M., \& Freund, J. B. 2015 Very near-nozzle shear-layer turbulence and jet noise. J. Fluid Mech. 770, 27-51.

Freund, J. B. 1997 Proposed inflow/outflow boundary condition for direct computation of aerodynamic sound. AIAA J. 35 (4), 740-742.

Freund, J. B. 2001 Noise sources in a low-Reynolds-number turbulent jet at Mach 0.9. J. Fluid Mech. 438, 277-305.

Gottlieb, S. \& Shu, W. 1998 Mathematics of Computation 67 (221), 73-85.

Gudmundsson, K. \& Colonius, T. 2011 Instability wave models for the near field fluctuations of turbulent jets. J. Fluid Mech. 689, 97-128.

Hill, G., Jenkins, R. C. \& Gilbert, B. L. 1976 Effects of the initial boundary-layer state on turbulent jet mixing. AIAA J. 14 (11), 1513-1514.

Husain, Z. D. \& Hussain, A. K. M. F. 1979 Axisymmetric mixing layer: influence of the initial and boundary conditions. AIAA J. 17 (1), 48-55.

Hussain, A. K. M. F \& Zedan, M. F. $1978 a$ Effects of the initial condition on the axisymmetric free shear layer: Effects of the initial fluctuation level. Phys. Fluids 21(9), 1475-1481.

Hussain, A. K. M. F \& Zedan, M. F. $1978 b$ Effects of the initial condition on the axisymmetric free shear layer: Effects of the initial momentum thickness. Phys. Fluids 21(7), 1100-1112.

Jordan, P. \& Colonius, T. 2013 Wave packets and turbulent jet noise. Ann. Rev. Fluid Mech. 45, 173-95.

Juvé, D., Sunyach, M. \& Comte-Bellot, G. 1979 Filtered azimuthal correlations in the acoustic far field of a subsonic jet. AIAA J. (17), 112-113.

Karon, A. Z. \& Ahuja, K. K. 2013 Effect of nozzle-exit boundary layer on jet noise. AIAA paper 2013-0615.

KaWAi, S. \& Larsson, J. 2012 Wall-modeling in large eddy simulation: Length scales, grid resolution, and accuracy. Phys. Fluids 24(015105).

Klein, M., SAdiki, A. \& JAnickA, J. 2003 A digital filter based generation of inflow data for spatially developing direct numerical or large eddy simulations. J. Comput. Phys. 186 (2), 652-665.

Kopiev, V., Chernyshev, S., Faranosov, G., Zaitsev, M. \& Belayev, I. 2010 Correlations of jet noise azimuthal components and their role in source identification. AIAA paper 20104018.

Larsson, J., Kawai, S., Bodart, J. \& Bermejo-Moreno, I. 2016 Large eddy simulation with modeled wall-stress: recent progress and future directions. JSME Mech. Eng. Reviews 3. 
Lesshafft, L. \& Huerre, P. 2007 Linear impulse response in hot round jets. Phys. Fluids 19, 024102 .

LockARD, D. P. 2000 An efficient, two-dimensional implementation of the Ffowcs Williams and Hawkings equation. J. Sound Vib. 229, 897-911.

Lorteau, M., Cléro, F. \& Vuillot, F. 2015 Analysis of noise radiation mechanisms in a hot subsonic jet from a validated large eddy simulation solution. Phys. Fluids 27, 075108.

Mani, A. 2012 Analysis and optimization of numerical sponge layers as a nonreflective boundary treatment. Journal of Computational Physics 231, 704-7016.

Mendez, S., Shoeybi, M., Sharma, A., Ham, F. E., Lele, S. K. \& Moin, P. 2012 Largeeddy simulations of perfectly-expanded supersonic jets using an unstructured solver. AIAA J. 50 (5), 1103-1118.

Michalke, A. 1984 Survey on jet instability theory. Progress in Aerospace Sciences 21, 159199.

Michalke, A. \& Fuchs, H. V. 1975 On turbulence and noise of an axisymmetric shear flow. J. Fluid Mech. 70, 179-205.

Morris, P. J., Long, L. N., Scheidegger, T. E. \& Bolurianan, S. 2002 Simulations of supersonic jet noise. International Journal of Aeroacoustics 1 (1), $17-41$.

Petersen, R. A. \& Samet, M. M. 1988 On the preferred mode of jet instability. Journal of Fluid Mechanics 194 (1), 153-173.

Piomelli, U. \& Balaras, E. 2002 Wall-layer models for large-eddy simulations. Ann. Rev. Fluid Mech. 34, 349-374.

Pounngué, A. Fosso, Sanjosé, M. \& Moreau, S. 2012 Jet noise simulation with realistic nozzle geometries using fully unstructured LES solver. AIAA paper 2012-2190.

Sandberg, R. D., Sandham, N. D. \& Suponitsky, V. 2012 DNS of a compressible pipe flow exiting into a coflow. Int. J. Heat and Fluid Flow 35, 33-44.

Sasaki, K., Cavalieri, A. V. G, Jordan, P., Schmidt, O. T., Colonius, T. \& Brès, G. A. 2017 High-frequency wavepackets in turbulent jets. J. Fluid Mech. 830.

Scarano, F. 2002 Iterative image deformation methods in piv. Meas. Sci. Tech. 13.

Schlichting, H. \& Gertsen, K. 2000 Boundary Layer Theory, 8th edn. Springer.

Schmidt, O. T., Towne, A., Colonius, T., Cavalieri, A. V. G., Jordan, P. \& Brès, G. A. 2017 Wavepackets and trapped acoustic modes in a turbulent jet: coherent structure eduction and global stability. J. Fluid Mech. 825, 1153-1181.

Shur, M. L., Spalart, P. R. \& Strelets, M. K. 2005 a Noise prediction for increasingly complex jets. part I: Methods and tests. Int. J. Aeroacoust. 4 (3-4), 213-246.

Shur, M. L., Spalart, P. R. \& Strelets, M. K. $2005 b$ Noise prediction for increasingly complex jets. part II: Applications. Int. J. Aeroacoust. 4 (3-4), 247-266.

Shur, M. L., Spalart, P. R. \& Strelets, M. K. 2011 Noise prediction for underexpanded jets in static and flight conditions. AIAA J. 49 (9), 2000-2017.

Spalart, P. R. 2009 Detached-eddy simulation. Ann. Rev. Fluid Mech. 41, 181-202.

Suponitsky, V., Sandham, N. D. \& Morfey, C. L. 2010 Linear and nonlinear mechanisms of sound radiation by instability waves in subsonic jet. J. Fluid Mech. 658, 509-538.

Suzuki, T. \& Colonius, T. 2006 Instability waves in a subsonic round jet detected using a near-field phased microphone array. Journal of Fluid Mechanics 565, 197-226.

Towne, A., Cavalieri, A. V. G., Jordan, P., Colonius, T., Schmidt, O., Jaunet, V. \& BrÈs, G. A. 2017 Acoustic resonance in the potential core of subsonic jets. J. Fluid Mech. 825, 1113-1152.

Trefethen, L. N. 2000 Spectral methods in MATLAB, , vol. 10. Society for Industrial Mathematics.

Uzun, A. \& Hussaini, Y. M. 2007 Investigation of high frequency noise generation in the near-nozzle region of a jet using large eddy simulation. Theor. Comput. Fluid Dyn. 21(4), 291-321.

Uzun, A., Lyrintsis, A. S. \& Blaisdell, G. A. 2004 Coupling of integral acoustics methods with LES for jet noise prediction. International Journal of Aeroacoustics 3(4), 297-346.

VReman, A. 2004 An eddy-viscosity subgrid-scale model for turbulent shear flow: Algebraic theory and applications. Physics of Fluids 16, 3570.

Vuillot, F., Lupoglazoff, N., Lorteau, M. \& Cléro, F. 2016 Large eddy simulation of 
jet noise from unstructured grids with turbulent nozzle boundary layer. AIAA paper 20163046.

Westerweel, Jerry \& Scarano, Fulvio 2005 Universal outlier detection for piv data. Experiments in Fluids 39 (6), 1096-1100.

WiEnEKe, B. 2005 Stereo-piv using self-calibration on particle images. Experiments in Fluids 39, $267-280$.

Zaman, K. B. M. Q. 1985 Effect of initial condition on subsonic jet noise. AIAA J. 23(9), $1370-1373$.

ZAman, K. B. M. Q. 1998 Asymptotic spreading rates of initially compressible jets - experiment and analysis. Phys. Fluids 10(10), 2652-2660.

ZAMAN, K. B. M. Q. 1999 Spreading characteristics of compressible jets from nozzles of various geometries. J. Fluid Mech. 383, 197-228.

Zaman, K. B. M. Q. 2012 Effect of initial boundary-layer state on subsonic jet noise. AIAA J. 50(8), 1784-1795. 